\title{
Numerical algorithms for multidimensional time-fractional wave equation of distributed-order with a nonlinear source term
}

\author{
Jiahui $\mathrm{Hu}^{1,2}$, Jungang Wang ${ }^{1}$ and Yufeng $\mathrm{Nie}^{\mathrm{e}^{*}}$
}

"Correspondence:

yfnie@nwpu.edu.cn

${ }^{1}$ Research Center for Computational

Science, Northwestern

Polytechnical University, Xi'an, China

Full list of author information is

available at the end of the article

\section{第 Springer}

\begin{abstract}
Fractional differential equations (FDEs) of distributed-order are important in depicting the models where the order of differentiation distributes over a certain range. Numerically solving this kind of FDEs requires not only discretizations of the temporal and spatial derivatives, but also approximation of the distributed-order integral, which brings much more difficulty. In this paper, based on the mid-point quadrature rule and composite two-point Gauss-Legendre quadrature rule, two finite difference schemes are established. Different from the previous works, which concerned only one- or two-dimensional problems with linear source terms, time-fractional wave equations of distributed-order whose source term is nonlinear in two and even three dimensions are considered. In addition, to improve the computational efficiency, the technique of alternating direction implicit (ADI) decomposition is also adopted. The unique solvability of the difference scheme is discussed, and the unconditional stability and convergence are analyzed. Finally, numerical experiments are carried out to verify the effectiveness and accuracy of the algorithms for both the two- and three-dimensional cases.
\end{abstract}

MSC: $35 \mathrm{R} 11 ; 65 \mathrm{M} 06 ; 65 \mathrm{M} 12$

Keywords: Time-fractional wave equation of distributed-order; ADI finite difference scheme; Nonlinear source term; Stability; Convergence

\section{Introduction}

The idea of distributed-order differential equation was first presented by Caputo for modeling the stress-strain behavior of an anelastic medium in [5] in the 1960s. Unlike the differential equations with the single-order fractional derivative and those with sums of fractional derivatives, i.e., multi-term FDEs, the distributed-order differential equations are derived by integrating the order of differentiation over a given range [1]. This can be regarded as a generalization of the aforementioned two classes of FDEs. Such FDEs are typically applied in a retarding sub-diffusion process, during which a plume of particles spreads at a logarithmic rate, and eventually the ultraslow diffusion is generated (see $[8$, $22,29])$. For example, the fractional Langevin equation of distributed-order was originally proposed for modeling the kinetics of retarding sub-diffusion, and then was found effi-

(c) The Author(s) 2018. This article is distributed under the terms of the Creative Commons Attribution 4.0 International License (http://creativecommons.org/licenses/by/4.0/), which permits unrestricted use, distribution, and reproduction in any medium, provided you give appropriate credit to the original author(s) and the source, provide a link to the Creative Commons license, and indicate if changes were made. 
cient in simulating the strongly anomalous ultraslow diffusion, where the mean square displacement grew as a power of logarithm of time [11]. Also, for optimal control problems, the integer order derivative is replaced by that of a distributed-order so as to capture delays of distinct sources. In [35], considering the optimal control problems with dynamics described by ordinary distributed-order fractional differential equations, the generalized necessary conditions were derived. Then an efficient numerical scheme was proposed and applied to solve an unconstrained convex distributed optimal control problem which is governed by the distributed-order fractional differential equations. In [34], a general formulation for the fractional optimal control problems with distributed-order fractional derivatives was presented, and a Legendre spectral collocation scheme for solving the derived boundary value problem was presented along with error analysis. Besides, there are also other various research fields involving the distributed-order FDEs, such as control and signal processing [20], modeling dielectric induction and diffusion [6], identification of systems [19], and so forth.

Recently, there have been many important progresses in the research of analytical solutions of distributed-order FDEs. For researching the kinetic description of anomalous diffusion and relaxation phenomena, Chechkin et al. [7] presented a diffusion-like equation with time fractional derivative of distributed-order, the authors also proved the positivity of the solutions and established the relation to the continuous-time random walk theory. Atanackovic et al. analyzed a Cauchy problem for a time distributed-order diffusion-wave equation by means of the theory of an abstract Volterra equation in [2]. In view of the fundamental solution of the Cauchy problem of the one-dimensional distributed-order diffusion-wave equation, Gorenflo et al. gave the interpretation that it is a probability density function of the space variable $x$ evolving in time $t$ in the transform domain by employing the technique of the Fourier and Laplace transforms [18]. Using the Laplace transform method, Li et al. [23] investigated the asymptotic behavior of solutions to the initial-boundary-value problem for the distributed-order time-fractional diffusion equations.

However, in most instances, analytical solutions of distributed-order differential equations are not readily available, thus researchers are stimulated to develop numerical algorithms for approximate solutions. To numerically solve the distributed-order differential equations, the distributed-order fractional derivatives are reduced to a sum of multi-term fractional derivatives by using a quadrature rule, then the multi-term fractional differential equations are left to be solved. In fact, the multi-term fractional differential equations can be regarded as a particular case of the distributed-order differential equations. To the best of our knowledge, the research on numerical solution of such fractional equations is still in its infancy. The references [10,21, 25] developed numerical methods for solving distributed-order ordinary differential equations. The authors of [3] constructed an efficient spectral method for solving multi-term time-space fractional partial differential equations by presenting a shifted Jacobi tau method for both temporal and spatial discretizations. A Legendre spectral tau method was employed for solving the multi-term time-fractional diffusion equations in [33], and the error estimation and convergence analysis was performed rigorously. Ford et al. developed an implicit finite difference method for the solution of the diffusion equation with distributed order in time [12]. By using the Grünwald-Letnikov formula, Gao et al. proposed two difference schemes to solve the one-dimensional distributed-order differential equations, and the extrapolation method 
was applied to improve the approximate accuracy [17]. In [13], the authors handled the one- and two-dimensional distributed-order diffusion equations by employing a weighted and shifted Grünwald-Letnikov formula to derive several high-order difference schemes. A distributed-order time and Riesz space-fractional Schrödinger equation was simulated by developing a new numerical approach in [4]. The considered problem was first transformed into a system of distributed-order fractional differential equations by employing the Jacobi-Gauss-Lobatto collocation method, and then the derived system was solved by a spectral method based on Jacobi-Gauss-Radau collocation method. In terms of the time distributed-order diffusion-wave equations, most of the work considers the onedimensional case, and the integrating range of the order of time derivative is the interval $[0,1]$, which is named the time distributed-order diffusion equation. When the order of the time derivative is distributed over the interval $[1,2]$, it is called the time distributedorder wave equation. Ye et al. derived and analyzed a compact difference scheme for a distributed-order time-fractional wave equation in [32].

In this work, we propose efficient finite difference schemes for solving the twoand three-dimensional time-fractional wave equations of distributed-order, respectively, where a nonlinear source term is considered. As far as we know, the relevant literature is rather limited. Gao et al. investigated ADI difference schemes for two-dimensional time distributed-order diffusion equations [14, 16]. In [14], Grünwald formula was employed and ADI difference scheme as well as compact ADI difference scheme were derived. The extrapolation method was also applied to obtain the improved approximate accuracy. In [16], the authors proposed two ADI difference schemes and proved that they were unconditionally stable and convergent in a discrete $L^{1}\left(L^{\infty}\right)$ norm. Based on the weighted and shifted Grünwald-Letnikov formula, they also developed two ADI difference schemes for solving the two-dimensional time distributed-order wave equations [15]. Abbaszadeh et al. solved the two-dimensional distributed order time-fractional diffusion-wave equation by combining the ADI approach with the interpolating element-free Galerkin method, where the time derivatives was discretized by a finite difference scheme [1]. Using the same time approximation method, and based on the shape functions of reproducing kernel particle method, a novel element-free Galerkin approach was proposed for solving 2D fractional Tricomi-type equation with Robin boundary condition [9]. Additionally, realizing the widespread use of the differential equations with nonlinear source terms [27, 31], Morgado et al. presented an implicit difference scheme for time distributed-order diffusion equation with a nonlinear source term in one dimension [24].

However, numerical approximations for multidimensional time distributed-order diffusion-wave equations with nonlinear source terms have not been considered yet, including two- and three-dimensional cases. In the current work, the multidimensional timefractional wave equation of distributed-order with a nonlinear source term is considered. To develop robust numerical algorithms, the mid-point quadrature rule and composite two-point Gauss-Legendre quadrature rule are used for the approximation of the distributed integral, respectively. As the multi-term time-fractional wave equation is left, the L1 formula is adopted for the discretization of the time-fractional derivatives. In the spatial direction, the central difference quotients are employed for approximating the secondorder derivatives. To linearize the nonlinear source term, the first order Taylor formula is used, whose advantage is making the computing procedure relatively simple. Necessarily, the technique of ADI decomposition is applied to improve the computational efficiency. 
The structure of the rest of this work is as follows. In Sect. 2, based on the mid-point quadrature rule and composite two-point Gauss-Legendre quadrature rule, two difference schemes for the two-dimensional problem are constructed and described in details. In Sect. 3, the solvability, stability and convergence of the derived difference schemes are discussed. Section 4 gives the description of ADI forms of the proposed schemes. We establish the ADI finite difference schemes for the three-dimensional problem in Sect. 5. Numerical results are illustrated in Sect. 6 to confirm the effectiveness and accuracy of our methods for both the two- and three-dimensional cases, and some conclusions are drawn in the last section.

\section{The derivation of the schemes for the two-dimensional problem}

We consider the two-dimensional problem first. The two-dimensional time-fractional wave equation of distributed-order with a nonlinear source term along with its initial and boundary conditions can be written as

$$
\begin{aligned}
& \int_{1}^{2} p(\beta)_{0}^{C} D_{t}^{\beta} u(x, y, t) d \beta=\frac{\partial^{2} u(x, y, t)}{\partial x^{2}}+\frac{\partial^{2} u(x, y, t)}{\partial y^{2}}+f(x, y, t, u(x, y, t)), \\
& \quad(x, y) \in \Omega, t \in(0, T], \\
& u(x, y, t)=\phi(x, y, t), \quad(x, y) \in \partial \Omega, t \in[0, T], \\
& u(x, y, 0)=\psi_{1}(x, y), \quad u_{t}(x, y, 0)=\psi_{2}(x, y), \quad(x, y) \in \Omega,
\end{aligned}
$$

where $\Omega=\left(0, L_{1}\right) \times\left(0, L_{2}\right)$, and $\partial \Omega$ is the boundary of $\Omega$. The fractional derivative ${ }_{0}^{C} D_{t}^{\beta} \nu(t)$ in (1) is given in the Caputo sense

$$
{ }_{0}^{C} D_{t}^{\beta} \nu(t)= \begin{cases}\frac{\partial v(t)}{\partial t}-\frac{\partial \nu(0)}{\partial t}, & \beta=1, \\ \frac{1}{\Gamma(2-\beta)} \int_{0}^{t}(t-\xi)^{1-\beta} \frac{\partial^{2} \nu(\xi)}{\partial \xi^{2}} d \xi, & 1<\beta<2, \\ \frac{\partial^{2} v(t)}{\partial t^{2}}, & \beta=2,\end{cases}
$$

the function $p(\beta)$ serves as a weight for the order of differentiation and is such that $p(\beta)>0$ and $\int_{1}^{2} p(\beta) d \beta=c_{0}>0$. We assume that $p(\beta), \phi(x, y, t), \psi_{1}(x, y), \psi_{2}(x, y)$ and $f(x, y, t, u)$ are continuous, and the nonlinear source term $f$ satisfies the Lipschitz condition with respect to $u$ :

$$
\left|f\left(x, y, t, u_{1}\right)-f\left(x, y, t, u_{2}\right)\right| \leq L_{f}\left|u_{1}-u_{2}\right|
$$

where $L_{f}$ is a positive constant.

The main procedure of developing numerical schemes for solving problem (1)-(3) is as follows. Firstly, a numerical quadrature rule is adopted to discretize the integral in (1), and a multi-term time-fractional wave equation is obtained in the process. Then the mid-point quadrature rule and the composite two-point Gauss-Legendre quadrature rule are employed for the discretization of the distributed integral, respectively. Secondly, we develop finite difference schemes which are uniquely solvable for the multi-term time-fractional wave equations, where the central difference quotient and the L1 formula are employed, and the Taylor formula is adopted for dealing with the nonlinear source term. Finally, by 
using the discrete energy method, we prove that the derived numerical schemes are unconditionally stable and convergent in the discrete $L^{2}$ norm, and then the ADI forms of the proposed schemes are given for computing.

The rest of this section focuses on deriving the finite difference scheme for the problem (1)-(3).

Let $M_{1}, M_{2}$ and $N$ be positive integers, and $h_{1}=L_{1} / M_{1}, h_{2}=L_{2} / M_{2}$ and $\tau=T / N$ denote the uniform sizes of spatial grid and time step, respectively. Then a spatial and temporal partition can be defined as $x_{i}=i h_{1}$ for $i=0,1, \ldots, M_{1}, y_{j}=j h_{2}$ for $j=0,1, \ldots, M_{2}$ and $t_{n}=n \tau$ for $n=0,1, \ldots, N$. Denote

$$
\begin{aligned}
& \Omega_{h}=\left\{\left(x_{i}, y_{j}\right) \mid 1 \leq i \leq M_{1}-1,1 \leq j \leq M_{2}-1\right\}, \\
& \partial \Omega_{h}=\left\{\left(x_{i}, y_{j}\right) \mid i=0 \text { or } i=M_{1} \text { or } j=0 \text { or } j=M_{2}\right\}, \quad \bar{\Omega}_{h}=\Omega_{h} \cup \partial \Omega_{h}, \\
& \Omega_{\tau}=\left\{t_{n} \mid t_{n}=n \tau, 0 \leq n \leq N\right\} .
\end{aligned}
$$

Then the domain $\bar{\Omega} \times[0, T]$ is covered by $\bar{\Omega}_{h} \times \Omega_{\tau}$. Let $u=\left\{u_{i j}^{n} \mid 0 \leq i \leq M_{1}, 0 \leq j \leq\right.$ $\left.M_{2}, 0 \leq n \leq N\right\}$ represent a grid function on $\bar{\Omega}_{h} \times \Omega_{\tau}$. We introduce the following notations:

$$
\begin{array}{ll}
u_{i j}^{n-\frac{1}{2}}=\frac{1}{2}\left(u_{i j}^{n}+u_{i j}^{n-1}\right), & \delta_{t} u_{i j}^{n-\frac{1}{2}}=\frac{1}{\tau}\left(u_{i j}^{n}-u_{i j}^{n-1}\right), \\
\delta_{x} u_{i-\frac{1}{2}, j}^{n}=\frac{1}{h_{1}}\left(u_{i j}^{n}-u_{i-1, j}^{n}\right), & \delta_{x}^{2} u_{i j}^{n}=\frac{1}{h_{1}}\left(\delta_{x} u_{i+\frac{1}{2}, j}^{n}-\delta_{x} u_{i-\frac{1}{2}, j}^{n}\right), \\
\delta_{y} u_{i, j-\frac{1}{2}}^{n}=\frac{1}{h_{2}}\left(u_{i j}^{n}-u_{i, j-1}^{n}\right), & \delta_{y}^{2} u_{i j}^{n}=\frac{1}{h_{2}}\left(\delta_{x} u_{i, j+\frac{1}{2}}^{n}-\delta_{x} u_{i, j-\frac{1}{2}}^{n}\right),
\end{array}
$$

and

$$
\Delta_{h} u_{i j}=\delta_{x}^{2} u_{i j}+\delta_{y}^{2} u_{i j}
$$

Consider Eq. (1) at the point $\left(x_{i}, y_{j}, t_{n}\right)$, which can be written as

$$
\begin{aligned}
& \int_{1}^{2} p(\beta)_{0}^{C} D_{t}^{\beta} u\left(x_{i}, y_{j}, t_{n}\right) d \beta \\
& \quad=\frac{\partial^{2} u\left(x_{i}, y_{j}, t_{n}\right)}{\partial x^{2}}+\frac{\partial^{2} u\left(x_{i}, y_{j}, t_{n}\right)}{\partial y^{2}}+f\left(x_{i}, y_{j}, t_{n}, u\left(x_{i}, y_{j}, t_{n}\right)\right) .
\end{aligned}
$$

Taking an average of Eq. (5) at time instants $t=t_{n}$ and $t=t_{n-1}$, we obtain the following equations:

$$
\begin{aligned}
\frac{1}{2}( & \left.\int_{1}^{2} p(\beta)_{0}^{C} D_{t}^{\beta} u\left(x_{i}, y_{j}, t_{n}\right) d \beta+\int_{1}^{2} p(\beta)_{0}^{C} D_{t}^{\beta} u\left(x_{i}, y_{j}, t_{n-1}\right) d \beta\right) \\
= & \frac{1}{2}\left[\frac{\partial^{2} u\left(x_{i}, y_{j}, t_{n}\right)}{\partial x^{2}}+\frac{\partial^{2} u\left(x_{i}, y_{j}, t_{n-1}\right)}{\partial x^{2}}\right]+\frac{1}{2}\left[\frac{\partial^{2} u\left(x_{i}, y_{j}, t_{n}\right)}{\partial y^{2}}+\frac{\partial^{2} u\left(x_{i}, y_{j}, t_{n-1}\right)}{\partial y^{2}}\right] \\
& +\frac{1}{2}\left[f\left(x_{i}, y_{j}, t_{n}, u\left(x_{i}, y_{j}, t_{n}\right)\right)+f\left(x_{i}, y_{j}, t_{n-1}, u\left(x_{i}, y_{j}, t_{n-1}\right)\right)\right] .
\end{aligned}
$$


Denote by $U_{i j}^{n}=u\left(x_{i}, y_{j}, t_{n}\right)$ the grid functions on $\bar{\Omega}_{h} \times \Omega_{\tau}$ with $0 \leq i \leq M_{1}, 0 \leq j \leq M_{2}$, $0 \leq n \leq N$. Equation (6) can be expressed as

$$
\begin{aligned}
\int_{1}^{2} p(\beta)_{0}^{C} D_{t}^{\beta} U_{i j}^{n-\frac{1}{2}} d \beta= & \frac{\partial^{2}}{\partial x^{2}} U_{i j}^{n-\frac{1}{2}}+\frac{\partial^{2}}{\partial y^{2}} U_{i j}^{n-\frac{1}{2}} \\
& +\frac{1}{2}\left[f\left(x_{i}, y_{j}, t_{n}, U_{i j}^{n}\right)+f\left(x_{i}, y_{j}, t_{n-1}, U_{i j}^{n-1}\right)\right] .
\end{aligned}
$$

Firstly, we discretize the integral term in (7). Here the mid-point quadrature rule and the composite two-point Gauss-Legendre quadrature rule are employed, respectively. Suppose $p(\beta) \in \mathcal{C}^{4}[1,2],\left.{ }_{0}^{C} D_{t}^{\beta} u\left(x_{i}, y_{j}, t\right)\right|_{t=t_{n-1}}$ and $\left.{ }_{0}^{C} D_{t}^{\beta} u\left(x_{i}, y_{j}, t\right)\right|_{t=t_{n}} \in \mathcal{C}^{4}[1,2]$. Let $K$ be a positive integer, and $\Delta \beta=1 / K$ denote the uniform step size. Take $\beta_{l}=1+\frac{2 l-1}{2} \Delta \beta$, $\widetilde{\beta}_{l-1}=1+(l-1) \Delta \beta, 1 \leq l \leq K$, and $\widetilde{\beta}_{K}=2$. When the mid-point quadrature rule is used for approximating the integral in (7), we are left with the following multi-term time-fractional wave equation:

$$
\begin{aligned}
\Delta \beta \sum_{l=1}^{K} p\left(\beta_{l}\right)_{0}^{C} D_{t}^{\beta_{l}} U_{i j}^{n-\frac{1}{2}}+R_{1}= & \frac{\partial^{2}}{\partial x^{2}} U_{i j}^{n-\frac{1}{2}}+\frac{\partial^{2}}{\partial y^{2}} U_{i j}^{n-\frac{1}{2}}+\frac{1}{2}\left[f\left(x_{i}, y_{j}, t_{n}, U_{i j}^{n}\right)\right. \\
& \left.+f\left(x_{i}, y_{j}, t_{n-1}, U_{i j}^{n-1}\right)\right]
\end{aligned}
$$

where $R_{1}=\mathcal{O}\left(\Delta \beta^{2}\right)$. While using the composite two-point Gauss-Legendre quadrature rule, we arrive at an analogous equation as follows:

$$
\begin{aligned}
& \frac{\Delta \beta}{2} \sum_{l=1}^{K}\left(p\left(\widetilde{\beta}_{l}^{(1)}\right)_{0}^{C} D_{t}^{\widetilde{\beta}_{l}^{(1)}} U_{i j}^{n-\frac{1}{2}}+p\left(\widetilde{\beta}_{l}^{(2)}\right)_{0}^{C} D_{t}^{\widetilde{\beta}_{l}^{(2)}} U_{i j}^{n-\frac{1}{2}}\right)+{ }^{\mathrm{GL}} R_{1} \\
& \quad=\frac{\partial^{2}}{\partial x^{2}} U_{i j}^{n-\frac{1}{2}}+\frac{\partial^{2}}{\partial y^{2}} U_{i j}^{n-\frac{1}{2}}+\frac{1}{2}\left[f\left(x_{i}, y_{j}, t_{n}, U_{i j}^{n}\right)+f\left(x_{i}, y_{j}, t_{n-1}, U_{i j}^{n-1}\right)\right],
\end{aligned}
$$

where $\widetilde{\beta}_{l}^{(1)}=\frac{\widetilde{\beta}_{l}+\widetilde{\beta}_{l-1}}{2}-\frac{\widetilde{\beta}_{l}-\widetilde{\beta}_{l-1}}{2 \sqrt{3}}, \widetilde{\beta}_{l}^{(2)}=\frac{\widetilde{\beta}_{l}+\widetilde{\beta}_{l-1}}{2}+\frac{\widetilde{\beta}_{l}-\widetilde{\beta}_{l-1}}{2 \sqrt{3}}, 1 \leq l \leq K$, and ${ }^{\mathrm{GL}} R_{1}=\mathcal{O}\left(\Delta \beta^{4}\right)$.

Next, we solve Eq. (8) or (9) with the boundary condition (2) and initial conditions (3). Here, we present the procedure for (8), and the technique used for (9) is similar.

Suppose $u(x, y, t) \in \mathcal{C}_{x, y, t}^{4,4,3}(\bar{\Omega} \times[0, T])$. According to Theorem 8.2.5 in [30], the Caputo derivative ${ }_{0}^{C} D_{t}^{\beta_{l}} U_{i j}^{n-\frac{1}{2}}, 1<\beta_{l}<2$ has the fully discrete difference scheme

$$
\begin{aligned}
{ }_{0}^{C} D_{t}^{\beta_{l}} U_{i j}^{n-\frac{1}{2}}= & \frac{\tau^{1-\beta_{l}}}{\Gamma\left(3-\beta_{l}\right)}\left[a_{0}^{\left(\beta_{l}\right)} \delta_{t} U_{i j}^{n-\frac{1}{2}}-\sum_{k=1}^{n-1}\left(a_{n-k-1}^{\left(\beta_{l}\right)}-a_{n-k}^{\left(\beta_{l}\right)}\right) \delta_{t} U_{i j}^{k-\frac{1}{2}}\right. \\
& \left.-a_{n-1}^{\left(\beta_{l}\right)} \psi_{2}\left(x_{i}, y_{j}\right)\right]+R_{2}^{l},
\end{aligned}
$$

where

$$
a_{k}^{\left(\beta_{l}\right)}=(k+1)^{2-\beta_{l}}-k^{2-\beta_{l}}, \quad k=0,1,2, \ldots,
$$


and

$$
\begin{aligned}
\left|R_{2}^{l}\right| \leq & \frac{1}{\Gamma\left(3-\beta_{l}\right)}\left[\frac{2-\beta_{l}}{12}+\frac{2^{3-\beta_{l}}}{3-\beta_{l}}-\left(1+2^{1-\beta_{l}}\right)+\frac{1}{12}\right] \\
& \cdot \max _{0 \leq t \leq t_{n}}\left|\frac{\partial^{3} u\left(x_{i}, y_{j}, t\right)}{\partial t^{3}}\right| \tau^{3-\beta_{l}}, \quad l=1,2, \ldots, K .
\end{aligned}
$$

In the meantime, the central difference quotient is used to approximate the second order derivatives in (8). Then we obtain

$$
\begin{aligned}
& \Delta \beta \sum_{l=1}^{K} p\left(\beta_{l}\right) \frac{\tau^{1-\beta_{l}}}{\Gamma\left(3-\beta_{l}\right)}\left[a_{0}^{\left(\beta_{l}\right)} \delta_{t} U_{i j}^{n-\frac{1}{2}}-\sum_{k=1}^{n-1}\left(a_{n-k-1}^{\left(\beta_{l}\right)}-a_{n-k}^{\left(\beta_{l}\right)}\right) \delta_{t} U_{i j}^{k-\frac{1}{2}}\right. \\
& \left.\quad-a_{n-1}^{\left(\beta_{l}\right)} \psi_{2}\left(x_{i}, y_{j}\right)\right]+\sum_{l=1}^{K} \Delta \beta p\left(\beta_{l}\right) R_{2}^{l}+R_{1} \\
& =\delta_{x}^{2} U_{i j}^{n-\frac{1}{2}}+\delta_{y}^{2} U_{i j}^{n-\frac{1}{2}}+\frac{1}{2}\left[f\left(x_{i}, y_{j}, t_{n-1}, U_{i j}^{n-1}\right)+f\left(x_{i}, y_{j}, t_{n}, U_{i j}^{n}\right)\right]+R_{3},
\end{aligned}
$$

where $R_{3}=\mathcal{O}\left(h_{1}^{2}+h_{2}^{2}\right)$. Subsequently, the nonlinear source term is dealt with in the following manner to avoid a system of nonlinear equations when computing:

$$
f\left(x_{i}, y_{j}, t_{n}, U_{i j}^{n}\right)=f\left(x_{i}, y_{j}, t_{n-1}, U_{i j}^{n-1}\right)+\mathcal{O}(\tau)
$$

Substituting Eq. (13) into Eq. (12), we have

$$
\begin{aligned}
& \Delta \beta \sum_{l=1}^{K} p\left(\beta_{l}\right) \frac{\tau^{1-\beta_{l}}}{\Gamma\left(3-\beta_{l}\right)}\left[a_{0}^{\left(\beta_{l}\right)} \delta_{t} U_{i j}^{n-\frac{1}{2}}-\sum_{k=1}^{n-1}\left(a_{n-k-1}^{\left(\beta_{l}\right)}-a_{n-k}^{\left(\beta_{l}\right)}\right) \delta_{t} U_{i j}^{k-\frac{1}{2}}\right. \\
& \left.\quad-a_{n-1}^{\left(\beta_{l}\right)} \psi_{2}\left(x_{i}, y_{j}\right)\right] \\
& =\delta_{x}^{2} U_{i j}^{n-\frac{1}{2}}+\delta_{y}^{2} U_{i j}^{n-\frac{1}{2}}+f\left(x_{i}, y_{j}, t_{n-1}, U_{i j}^{n-1}\right)+R_{i j}^{n-\frac{1}{2}}+\widetilde{R}_{i j}^{n-\frac{1}{2}}
\end{aligned}
$$

where

$$
\begin{aligned}
R_{i j}^{n-\frac{1}{2}} & =-\sum_{l=1}^{K} \Delta \beta p\left(\beta_{l}\right) R_{2}^{l}-R_{1}+R_{3} \\
& =-\sum_{l=1}^{K} \Delta \beta p\left(\beta_{l}\right) R_{2}^{l}+\mathcal{O}\left(h_{1}^{2}+h_{2}^{2}\right)+\mathcal{O}\left(\Delta \beta^{2}\right)
\end{aligned}
$$

and

$$
\widetilde{R}_{i j}^{n-\frac{1}{2}}=\mathcal{O}(\tau)
$$


In the following, we discuss the necessary estimate of $R_{i j}^{n-\frac{1}{2}}$. From (11), we can deduce that there exists a positive constant $C_{1}$ such that

$$
\left|-\sum_{l=1}^{K} \Delta \beta p\left(\beta_{l}\right) R_{2}^{l}\right| \leq C_{1} \tau^{1+\frac{1}{2} \Delta \beta} \sum_{l=1}^{K} \Delta \beta p\left(\beta_{l}\right) .
$$

Since

$$
\sum_{l=1}^{K} \Delta \beta p\left(\beta_{l}\right) \sim \int_{1}^{2} p(\beta) d \beta=c_{0}
$$

we get

$$
\sum_{l=1}^{K} \Delta \beta p\left(\beta_{l}\right) \leq C_{2}
$$

where $C_{2}$ is a positive constant. Thus there is a positive constant $C_{3}$ such that

$$
\left|R_{i j}^{n-\frac{1}{2}}\right| \leq C_{3}\left(\tau^{1+\frac{1}{2} \Delta \beta}+h_{1}^{2}+h_{2}^{2}+\Delta \beta^{2}\right)
$$

Besides, Eq. (15) implies that

$$
\left|\widetilde{R}_{i j}^{n-\frac{1}{2}}\right| \leq C_{4} \tau
$$

where $C_{4}$ is a positive constant.

To establish an efficient numerical scheme, the procedure below is needed. Denote

$$
\mu=\Delta \beta \sum_{l=1}^{K} p\left(\beta_{l}\right) \frac{1}{\tau^{\beta_{l}} \Gamma\left(3-\beta_{l}\right)} .
$$

Since

$$
\begin{aligned}
& \Delta \beta \sum_{l=1}^{K} p\left(\beta_{l}\right) \frac{1}{\tau^{\beta_{l}} \Gamma\left(3-\beta_{l}\right)} \\
& \sim \int_{1}^{2} p(\beta) \frac{1}{\tau^{\beta} \Gamma(3-\beta)} d \beta \\
&=\frac{p\left(\beta^{*}\right)}{\Gamma\left(3-\beta^{*}\right)} \int_{1}^{2} \frac{1}{\tau^{\beta}} d \beta \\
&=\frac{p\left(\beta^{*}\right)}{\Gamma\left(3-\beta^{*}\right)} \frac{1-\tau}{\tau^{2}|\ln \tau|},
\end{aligned}
$$

it can be concluded that

$$
\mu=\frac{1}{\mathcal{O}\left(\tau^{2}|\ln \tau|\right)} .
$$


When $\tau$ is sufficiently small, $|\ln \tau| \leq C \tau^{-\varepsilon}$ holds for any positive and small $\varepsilon$. Therefore, the term $\mathcal{O}\left(\tau^{2}|\ln \tau|\right)$ is almost the same as $\mathcal{O}\left(\tau^{2}\right)$ when $\tau$ is sufficiently small. Adding the high order term

$$
\widehat{R}_{i j}^{n-\frac{1}{2}}=\frac{\tau}{4 \mu} \delta_{x}^{2} \delta_{y}^{2} \frac{U_{i j}^{n}-U_{i j}^{n-1}}{\tau}
$$

on both sides of (14), we have

$$
\begin{aligned}
\Delta \beta & \sum_{l=1}^{K} p\left(\beta_{l}\right) \frac{\tau^{1-\beta_{l}}}{\Gamma\left(3-\beta_{l}\right)}\left[a_{0}^{\left(\beta_{l}\right)} \delta_{t} U_{i j}^{n-\frac{1}{2}}-\sum_{k=1}^{n-1}\left(a_{n-k-1}^{\left(\beta_{l}\right)}-a_{n-k}^{\left(\beta_{l}\right)}\right) \delta_{t} U_{i j}^{k-\frac{1}{2}}\right. \\
& \left.-a_{n-1}^{\left(\beta_{l}\right)} \psi_{2}\left(x_{i}, y_{j}\right)\right]+\frac{\tau}{4 \mu} \delta_{x}^{2} \delta_{y}^{2} \frac{U_{i j}^{n}-U_{i j}^{n-1}}{\tau} \\
= & \delta_{x}^{2} U_{i j}^{n-\frac{1}{2}}+\delta_{y}^{2} U_{i j}^{n-\frac{1}{2}}+f\left(x_{i}, y_{j}, t_{n-1}, U_{i j}^{n-1}\right)+R_{i j}^{n-\frac{1}{2}}+\widetilde{R}_{i j}^{n-\frac{1}{2}}+\widehat{R}_{i j}^{n-\frac{1}{2}} .
\end{aligned}
$$

From the analysis of $\mu$, we get

$$
\left|\widehat{R}_{i j}^{n-\frac{1}{2}}\right| \leq C_{5} \tau^{3}|\ln \tau|
$$

where $C_{5}$ is a positive constant. Also, for the initial and boundary conditions, we have

$$
\begin{aligned}
& U_{i j}^{0}=\psi_{1}\left(x_{i}, y_{j}\right), \quad\left(x_{i}, y_{j}\right) \in \Omega_{h}, \\
& U_{i j}^{n}=\phi\left(x_{i}, y_{j}, t_{n}\right), \quad\left(x_{i}, y_{j}\right) \in \partial \Omega_{h}, 0 \leq n \leq N .
\end{aligned}
$$

Let $u_{i j}^{n}$ be a numerical approximation to $u\left(x_{i}, y_{j}, t_{n}\right)$. Neglecting the small terms $R_{i j}^{n-\frac{1}{2}}$, $\widetilde{R}_{i j}^{n-\frac{1}{2}}$ and $\widehat{R}_{i j}^{n-\frac{1}{2}}$ in (16), and using $u_{i j}^{n}$ instead of $U_{i j}^{n}$ in Eqs. (16)-(18), the finite difference scheme for (1)-(3) is developed as follows:

$$
\begin{aligned}
\Delta \beta & \sum_{l=1}^{K} p\left(\beta_{l}\right) \frac{\tau^{1-\beta_{l}}}{\Gamma\left(3-\beta_{l}\right)}\left[a_{0}^{\left(\beta_{l}\right)} \delta_{t} u_{i j}^{n-\frac{1}{2}}-\sum_{k=1}^{n-1}\left(a_{n-k-1}^{\left(\beta_{l}\right)}-a_{n-k}^{\left(\beta_{l}\right)}\right) \delta_{t} u_{i j}^{k-\frac{1}{2}}\right. \\
& \left.-a_{n-1}^{\left(\beta_{l}\right)}\left(\psi_{2}\right)_{i j}\right]+\frac{\tau}{4 \mu} \delta_{x}^{2} \delta_{y}^{2} \frac{u_{i j}^{n}-u_{i j}^{n-1}}{\tau} \\
= & \delta_{x}^{2} u_{i j}^{n-\frac{1}{2}}+\delta_{y}^{2} u_{i j}^{n-\frac{1}{2}}+f\left(x_{i}, y_{j}, t_{n-1}, u_{i j}^{n-1}\right), \\
& 1 \leq i \leq M_{1}-1,1 \leq j \leq M_{2}-1,1 \leq n \leq N, \\
u_{i j}^{0}= & \left(\psi_{1}\right)_{i j}, \quad 1 \leq i \leq M_{1}-1,1 \leq j \leq M_{2}-1, \\
u_{i j}^{n}= & \phi_{i j}^{n}, \quad(i, j) \in \gamma=\left\{(i, j) \mid\left(x_{i}, y_{j}\right) \in \partial \Omega_{h}\right\}, 0 \leq n \leq N,
\end{aligned}
$$

where

$$
\left(\psi_{1}\right)_{i j}=\psi_{1}\left(x_{i}, y_{j}\right), \quad\left(\psi_{2}\right)_{i j}=\psi_{2}\left(x_{i}, y_{j}\right), \quad 1 \leq i \leq M_{1}-1,1 \leq j \leq M_{2}-1,
$$


and

$$
\phi_{i j}^{n}=\phi\left(x_{i}, y_{j}, t_{n}\right), \quad(i, j) \in \gamma, 0 \leq n \leq N
$$

Starting the procedure by Eq. (9), we have the scheme for solving (1)-(3) as

$$
\begin{aligned}
\frac{\Delta \beta}{2} \sum_{l=1}^{K} p\left(\widetilde{\beta}_{l}^{(1)}\right) \frac{\tau^{1-\widetilde{\beta}_{l}^{(1)}}}{\Gamma\left(3-\widetilde{\beta}_{l}^{(1)}\right)}\left[a_{0}^{\left(\widetilde{\beta}_{l}^{(1)}\right)} \delta_{t} u_{i j}^{n-\frac{1}{2}}-\sum_{k=1}^{n-1}\left(a_{n-k-1}^{\left(\widetilde{\beta}_{l}^{(1)}\right)}-a_{n-k}^{\left(\widetilde{\beta}_{l}^{(1)}\right)}\right) \delta_{t} u_{i j}^{k-\frac{1}{2}}\right. \\
\left.\quad-a_{n-1}^{\left(\widetilde{\beta}_{l}^{(1)}\right)}\left(\psi_{2}\right)_{i j}\right]+\frac{\Delta \beta}{2} \sum_{l=1}^{K} p\left(\widetilde{\beta}_{l}^{(2)}\right) \frac{\tau^{1-\widetilde{\beta}_{l}^{(2)}}}{\Gamma\left(3-\widetilde{\beta}_{l}^{(2)}\right)}\left[a_{0}^{\left(\widetilde{\beta}_{l}^{(2)}\right)} \delta_{t} u_{i j}^{n-\frac{1}{2}}\right. \\
\left.\quad-\sum_{k=1}^{n-1}\left(a_{n-k-1}^{\left(\widetilde{\beta}_{l}^{(2)}\right)}-a_{n-k}^{\left(\widetilde{\beta}_{l}^{(2)}\right)}\right) \delta_{t} u_{i j}^{k-\frac{1}{2}}-a_{n-1}^{\left(\widetilde{\beta}_{l}^{(2)}\right)}\left(\psi_{2}\right)_{i j}\right]+\frac{\tau}{4 \widetilde{\mu}} \delta_{x}^{2} \delta_{y}^{2} \frac{u_{i j}^{n}-u_{i j}^{n-1}}{\tau} \\
=\delta_{x}^{2} u_{i j}^{n-\frac{1}{2}}+\delta_{y}^{2} u_{i j}^{n-\frac{1}{2}}+f\left(x_{i}, y_{j}, t_{n-1}, u_{i j}^{n-1}\right), \\
\quad 1 \leq i \leq M_{1}-1,1 \leq j \leq M_{2}-1,1 \leq n \leq N, \\
u_{i j}^{0}=\left(\psi_{1}\right)_{i j}, \quad 1 \leq i \leq M_{1}-1,1 \leq j \leq M_{2}-1, \\
u_{i j}^{n}=\phi_{i j}^{n}, \quad(i, j) \in \gamma, 0 \leq n \leq N,
\end{aligned}
$$

where the omitted remainder is $\mathcal{O}\left(\tau+h_{1}^{2}+h_{2}^{2}+\Delta \beta^{4}\right)$, and

$$
\tilde{\mu}=\frac{\Delta \beta}{2} \sum_{l=1}^{K} p\left(\widetilde{\beta}_{l}^{(1)}\right) \frac{1}{\tau^{\widetilde{\beta}_{l}^{(1)}} \Gamma\left(3-\widetilde{\beta}_{l}^{(1)}\right)}+\frac{\Delta \beta}{2} \sum_{l=1}^{K} p\left(\widetilde{\beta}_{l}^{(2)}\right) \frac{1}{\tau^{\widetilde{\beta}_{l}^{(2)}} \Gamma\left(3-\widetilde{\beta}_{l}^{(2)}\right)} .
$$

\section{Analysis of the difference scheme}

In this section, the analysis of scheme (19)-(21) is carried out, and that for scheme (22)(24) is similar.

\subsection{Unique solvability}

In this subsection, the unique solvability of scheme (19)-(21) is proved.

Theorem 1 The finite difference scheme (19)-(21) is uniquely solvable.

Proof Let $u^{n}=\left\{u_{i j}^{n} \mid 0 \leq i \leq M_{1}, 0 \leq j \leq M_{2}\right\}$. It can be seen that the value of $u^{0}$ is uniquely determined by (20) and (21). Suppose the values of $u^{0}, u^{1}, \ldots, u^{n-1}$ have been uniquely determined. To show that the linear system of equations (19) and (21) has a unique solution, it is sufficient to prove that the corresponding homogeneous one, namely

$$
\begin{aligned}
& \mu u_{i j}^{n}-\frac{1}{2} \Delta_{h} u_{i j}^{n}+\frac{1}{4 \mu} \delta_{x}^{2} \delta_{y}^{2} u_{i j}^{n}=0, \quad 1 \leq i \leq M_{1}-1,1 \leq j \leq M_{2}-1, \\
& u_{i j}^{n}=0, \quad i=0 \text { or } i=M_{1} \text { or } j=0 \text { or } j=M_{2}
\end{aligned}
$$

only has the zero solution. 
Multiplying (25) by $h_{1} h_{2} u_{i j}^{n}$, summing over $i$ from 1 to $M_{1}-1$ and over $j$ from 1 to $M_{2}-1$, we have

$$
\mu\left\|u^{n}\right\|^{2}+\frac{1}{2}\left|u^{n}\right|^{2}+\frac{1}{4 \mu}\left\|\delta_{x} \delta_{y} u^{n}\right\|^{2}=0 .
$$

Equation (27) implies $\left\|u^{n}\right\|=0$. Combining with (26) this yields $u^{n}=0$.

According to the principle of mathematical induction, the proof is completed.

\subsection{Stability}

In this subsection we focus on showing the unconditional stability of the difference scheme (19)-(21). We introduce some auxiliary definitions and useful results which will also be used when the convergence is considered.

Denote the space of grid functions on $\bar{\Omega}_{h}$ by

$$
\mathcal{V}_{h}=\left\{v \mid v=\left\{v_{i j} \mid\left(x_{i}, y_{j}\right) \in \bar{\Omega}_{h}\right\} \text { and } v_{i j}=0 \text { if }\left(x_{i}, y_{j}\right) \in \partial \Omega_{h}\right\}
$$

For any grid function $v \in \mathcal{V}_{h}$, the following discrete norms and Sobolev seminorm are introduced:

$$
\begin{aligned}
& \|v\|=\sqrt{h_{1} h_{2} \sum_{i=1}^{M_{1}-1} \sum_{j=1}^{M_{2}-1}\left|v_{i j}\right|^{2},} \quad\left\|\delta_{x} \delta_{y} v\right\|=\sqrt{h_{1} h_{2} \sum_{i=1}^{M_{1}} \sum_{j=1}^{M_{2}}\left|\delta_{x} \delta_{y} v_{i-\frac{1}{2}, j-\frac{1}{2}}\right|^{2}}, \\
& \left\|\delta_{x} v\right\|=\sqrt{h_{1} h_{2} \sum_{i=1}^{M_{1}} \sum_{j=1}^{M_{2}-1}\left|\delta_{x} v_{i-\frac{1}{2}, j}\right|^{2},} \quad\left\|\delta_{y} v\right\|=\sqrt{h_{1} h_{2} \sum_{i=1}^{M_{1}-1} \sum_{j=1}^{M_{2}}\left|\delta_{y} v_{i, j-\frac{1}{2}}\right|^{2}}, \\
& \left\|\Delta_{h} v\right\|=\sqrt{h_{1} h_{2} \sum_{i=1}^{M_{1}-1} \sum_{j=1}^{M_{2}-1}\left|\Delta_{h} v_{i j}\right|^{2},} \quad|v|_{1}=\sqrt{\left\|\delta_{x} v\right\|^{2}+\left\|\delta_{y} v\right\|^{2}} .
\end{aligned}
$$

Lemma 1 ([28]) For any grid function $v \in \mathcal{V}_{h},\|v\| \leq \frac{L_{1} L_{2}}{\sqrt{6\left(L_{1}^{2}+L_{2}^{2}\right)}}|v|_{1}$.

Lemma 2 ([30]) For any grid function $v \in \mathcal{V}_{h},|v|_{1} \leq \frac{L_{1} L_{2}}{\sqrt{6\left(L_{1}^{2}+L_{2}^{2}\right)}}\left\|\Delta_{h} v\right\|$.

Lemma 3 ([30]) For any $G=\left\{G_{1}, G_{2}, G_{3}, \ldots\right\}$ and $q$, we have

$$
\begin{gathered}
\sum_{n=1}^{s}\left[b_{0} G_{n}-\sum_{k=1}^{n-1}\left(b_{n-k-1}-b_{n-k}\right) G_{k}-b_{n-1} q\right] G_{n} \\
\geq \frac{t_{s}^{1-\alpha}}{2} \tau \sum_{n=1}^{s} G_{n}^{2}-\frac{t_{s}^{2-\alpha}}{2(2-\alpha)} q^{2}, \quad s=1,2,3, \ldots
\end{gathered}
$$

where

$$
b_{l}=\frac{\tau^{2-\alpha}}{2-\alpha}\left[(l+1)^{2-\alpha}-l^{2-\alpha}\right], \quad l=0,1,2, \ldots
$$

The discrete Gronwall's inequality is also necessary and is stated below. 
Lemma 4 ([26]) Assume that $k_{n}$ and $p_{n}$ are nonnegative sequences, and the sequence $\Phi_{n}$ satisfies

$$
\Phi_{0} \leq g_{0}, \quad \Phi_{n} \leq g_{0}+\sum_{l=0}^{n-1} p_{l}+\sum_{l=0}^{n-1} k_{l} \Phi_{l}, \quad n \geq 1,
$$

where $g_{0} \geq 0$. Then the sequence $\Phi_{n}$ satisfies

$$
\Phi_{n} \leq\left(g_{0}+\sum_{l=0}^{n-1} p_{l}\right) \exp \left(\sum_{l=0}^{n-1} k_{l}\right), \quad n \geq 1 .
$$

Assume that $\widetilde{u}_{i j}^{n}$ is an approximation solution of $u_{i j}^{n}$, which is the exact solution of the scheme (19)-(21). Also, suppose that $\left(\widetilde{\psi}_{1}\right)_{i j}$ and $\left(\widetilde{\psi}_{2}\right)_{i j}$ are approximations to $\left(\psi_{1}\right)_{i j}$ and $\left(\psi_{2}\right)_{i j}$, respectively. Denote $\varepsilon_{i j}^{n}=u_{i j}^{n}-\widetilde{u}_{i j}^{n}, 0 \leq i \leq M_{1}, 0 \leq j \leq M_{2}, 0 \leq n \leq N$. Then we have the perturbation error equations

$$
\begin{aligned}
\Delta \beta & \sum_{l=1}^{K} p\left(\beta_{l}\right) \frac{\tau^{1-\beta_{l}}}{\Gamma\left(3-\beta_{l}\right)}\left[a_{0}^{\left(\beta_{l}\right)} \delta_{t} \varepsilon_{i j}^{n-\frac{1}{2}}-\sum_{k=1}^{n-1}\left(a_{n-k-1}^{\left(\beta_{l}\right)}-a_{n-k}^{\left(\beta_{l}\right)}\right) \delta_{t} \varepsilon_{i j}^{k-\frac{1}{2}}\right. \\
& \left.-a_{n-1}^{\left(\beta_{l}\right)}\left(\psi_{2}^{*}\right)_{i j}\right]+\frac{\tau}{4 \mu} \delta_{x}^{2} \delta_{y}^{2} \frac{\varepsilon_{i j}^{n}-\varepsilon_{i j}^{n-1}}{\tau} \\
= & \delta_{x}^{2} \varepsilon_{i j}^{n-\frac{1}{2}}+\delta_{y}^{2} \varepsilon_{i j}^{n-\frac{1}{2}}+f\left(x_{i}, y_{j}, t_{n-1}, u_{i j}^{n-1}\right)-f\left(x_{i}, y_{j}, t_{n-1}, \widetilde{u}_{i j}^{n-1}\right), \\
& 1 \leq i \leq M_{1}-1,1 \leq j \leq M_{2}-1,1 \leq n \leq N, \\
\varepsilon_{i j}^{0}= & \left(\psi_{1}\right)_{i j}-\left(\widetilde{\psi}_{1}\right)_{i j}, \quad 1 \leq i \leq M_{1}-1,1 \leq j \leq M_{2}-1, \\
\varepsilon_{i j}^{n}= & 0, \quad(i, j) \in \gamma, 0 \leq n \leq N,
\end{aligned}
$$

where $\left(\psi_{2}^{*}\right)_{i j}=\left(\psi_{2}\right)_{i j}-\left(\widetilde{\psi}_{2}\right)_{i j}$.

Theorem 2 Assume that condition (4) is satisfied. Then the difference scheme (19)-(21) is unconditionally stable.

Proof Let

$$
b_{k}^{\left(\beta_{l}\right)}=\frac{\tau^{2-\beta_{l}}}{2-\beta_{l}} a_{k}^{\left(\beta_{l}\right)}, \quad 1 \leq l \leq K .
$$

Then Eq. (28) is equivalent to

$$
\begin{gathered}
\Delta \beta \sum_{l=1}^{K} p\left(\beta_{l}\right) \frac{1}{\Gamma\left(2-\beta_{l}\right) \tau}\left[b_{0}^{\left(\beta_{l}\right)} \delta_{t} \varepsilon_{i j}^{n-\frac{1}{2}}-\sum_{k=1}^{n-1}\left(b_{n-k-1}^{\left(\beta_{l}\right)}-b_{n-k}^{\left(\beta_{l}\right)}\right) \delta_{t} \varepsilon_{i j}^{k-\frac{1}{2}}\right. \\
\left.-b_{n-1}^{\left(\beta_{l}\right)}\left(\psi_{2}^{*}\right)_{i j}\right]+\frac{\tau}{4 \mu} \delta_{x}^{2} \delta_{y}^{2} \frac{\varepsilon_{i j}^{n}-\varepsilon_{i j}^{n-1}}{\tau} \\
=\delta_{x}^{2} \varepsilon_{i j}^{n-\frac{1}{2}}+\delta_{y}^{2} \varepsilon_{i j}^{n-\frac{1}{2}}+f\left(x_{i}, y_{j}, t_{n-1}, u_{i j}^{n-1}\right)-f\left(x_{i}, y_{j}, t_{n-1}, \widetilde{u}_{i j}^{n-1}\right), \\
1 \leq i \leq M_{1}-1,1 \leq j \leq M_{2}-1,1 \leq n \leq N .
\end{gathered}
$$


Multiplying (29) by $h_{1} h_{2} \tau \delta_{t} \varepsilon_{i j}^{n-\frac{1}{2}}$, summing over $i$ from 1 to $M_{1}-1$, over $j$ from 1 to $M_{2}-1$, and over $n$ from 1 to $s$, we analyze each term in the resulted equation. Firstly, by Lemma 3, we have

$$
\begin{gathered}
\Delta \beta \sum_{l=1}^{K} p\left(\beta_{l}\right) \frac{1}{\Gamma\left(2-\beta_{l}\right)} h_{1} h_{2} \sum_{i=1}^{M_{1}-1} \sum_{j=1}^{M_{2}-1}\left\{\sum _ { n = 1 } ^ { s } \left[b_{0}^{\left(\beta_{l}\right)} \delta_{t} \varepsilon_{i j}^{n-\frac{1}{2}}\right.\right. \\
\left.\left.-\sum_{k=1}^{n-1}\left(b_{n-k-1}^{\left(\beta_{l}\right)}-b_{n-k}^{\left(\beta_{l}\right)}\right) \delta_{t} \varepsilon_{i j}^{k-\frac{1}{2}}-b_{n-1}^{\left(\beta_{l}\right)}\left(\psi_{2}^{*}\right)_{i j}\right] \delta_{t} \varepsilon_{i j}^{n-\frac{1}{2}}\right\} \\
\geq \Delta \beta \sum_{l=1}^{K} p\left(\beta_{l}\right) \frac{1}{\Gamma\left(2-\beta_{l}\right)}\left[\frac{1}{2} t_{s}^{1-\beta_{l}} \tau \sum_{n=1}^{s}\left\|\delta_{t} \varepsilon^{n-\frac{1}{2}}\right\|^{2}\right. \\
\left.\quad-\frac{t_{s}^{2-\beta_{l}}}{2\left(2-\beta_{l}\right)} h_{1} h_{2} \sum_{i=1}^{M_{1}-1} \sum_{j=1}^{M_{2}-1}\left(\psi_{2}^{*}\right)_{i j}^{2}\right] \\
=\frac{1}{2} \tau K_{s} \sum_{n=1}^{s}\left\|\delta_{t} \varepsilon^{n-\frac{1}{2}}\right\|^{2}-\Delta \beta \sum_{l=1}^{K} p\left(\beta_{l}\right) \frac{t_{s}^{2-\beta_{l}}}{2 \Gamma\left(3-\beta_{l}\right)}\left\|\psi_{2}^{*}\right\|^{2},
\end{gathered}
$$

where

$$
K_{s}=\Delta \beta \sum_{l=1}^{K} p\left(\beta_{l}\right) \frac{t_{s}^{1-\beta_{l}}}{\Gamma\left(2-\beta_{l}\right)}>0 .
$$

Subsequently, using the discrete Green formula, we get the following two equations:

$$
\begin{aligned}
h_{1} & h_{2} \tau \sum_{i=1}^{M_{1}-1} \sum_{j=1}^{M_{2}-1} \sum_{n=1}^{s} \frac{\tau}{4 \mu} \delta_{x}^{2} \delta_{y}^{2} \frac{\varepsilon_{i j}^{n}-\varepsilon_{i j}^{n-1}}{\tau} \delta_{t} \varepsilon_{i j}^{n-\frac{1}{2}} \\
= & \frac{1}{4 \mu} \sum_{n=1}^{s} h_{1} h_{2} \sum_{i=1}^{M_{1}} \sum_{j=1}^{M_{2}}\left(\delta_{x} \delta_{y}\left(\varepsilon_{i-\frac{1}{2}, j-\frac{1}{2}}^{n}-\varepsilon_{i-\frac{1}{2}, j-\frac{1}{2}}^{n-1}\right)\right)\left(\delta_{x} \delta_{y}\left(\varepsilon_{i-\frac{1}{2}, j-\frac{1}{2}}^{n}-\varepsilon_{i-\frac{1}{2}, j-\frac{1}{2}}^{n-1}\right)\right) \\
= & \frac{1}{4 \mu} \sum_{n=1}^{s}\left\|\delta_{x} \delta_{y}\left(\varepsilon^{n}-\varepsilon^{n-1}\right)\right\|^{2} \geq 0,
\end{aligned}
$$

and

$$
\begin{aligned}
\tau & \sum_{n=1}^{s}\left[h_{1} h_{2} \sum_{i=1}^{M_{1}-1} \sum_{j=1}^{M_{2}-1}\left(\delta_{t} \varepsilon_{i j}^{n-\frac{1}{2}}\right)\left(\delta_{x}^{2} \varepsilon_{i j}^{n-\frac{1}{2}}\right)\right] \\
& =-\tau \sum_{n=1}^{s}\left[h_{1} h_{2} \sum_{j=1}^{M_{2}-1} \sum_{i=1}^{M_{1}}\left(\delta_{x} \varepsilon_{i-\frac{1}{2}, j}^{n-\frac{1}{2}}\right)\left(\delta_{t} \delta_{x} \varepsilon_{i-\frac{1}{2}, j}^{n-\frac{1}{2}}\right)\right] \\
& =-\tau \sum_{n=1}^{s}\left[h_{1} h_{2} \sum_{j=1}^{M_{2}-1} \sum_{i=1}^{M_{1}}\left(\frac{\delta_{x} \varepsilon_{i-\frac{1}{2}, j}^{n}+\delta_{x} \varepsilon_{i-\frac{1}{2}, j}^{n-1}}{2}\right)\left(\frac{\delta_{x} \varepsilon_{i-\frac{1}{2}, j}^{n}-\delta_{x} \varepsilon_{i-\frac{1}{2}, j}^{n-1}}{\tau}\right)\right] \\
& =-\frac{1}{2}\left[\left\|\delta_{x} \varepsilon^{s}\right\|^{2}-\left\|\delta_{x} \varepsilon^{0}\right\|^{2}\right] .
\end{aligned}
$$


Analogous to (32), we also obtain

$$
\tau \sum_{n=1}^{s}\left[h_{1} h_{2} \sum_{i=1}^{M_{1}-1} \sum_{j=1}^{M_{2}-1}\left(\delta_{t} \varepsilon_{i j}^{n-\frac{1}{2}}\right)\left(\delta_{y}^{2} \varepsilon_{i j}^{n-\frac{1}{2}}\right)\right]=-\frac{1}{2}\left[\left\|\delta_{y} \varepsilon^{s}\right\|^{2}-\left\|\delta_{y} \varepsilon^{0}\right\|^{2}\right] .
$$

On the basis of (4), we have

$$
\begin{aligned}
& h_{1} h_{2} \sum_{i=1}^{M_{1}-1} \sum_{j=1}^{M_{2}-1}\left[\tau \sum_{n=1}^{s}\left(\delta_{t} \varepsilon_{i j}^{n-\frac{1}{2}}\right)\left(f\left(x_{i}, y_{j}, t_{n-1}, u_{i j}^{n-1}\right)-f\left(x_{i}, y_{j}, t_{n-1}, \tilde{u}_{i j}^{n-1}\right)\right)\right] \\
& \leq h_{1} h_{2} \sum_{i=1}^{M_{1}-1} \sum_{j=1}^{M_{2}-1}\left[\tau \sum_{n=1}^{s}\left|\delta_{t} \varepsilon_{i j}^{n-\frac{1}{2}}\right|\left|f\left(x_{i}, y_{j}, t_{n-1}, u_{i j}^{n-1}\right)-f\left(x_{i}, y_{j}, t_{n-1}, \tilde{u}_{i j}^{n-1}\right)\right|\right] \\
& \leq h_{1} h_{2} \sum_{i=1}^{M_{1}-1} \sum_{j=1}^{M_{2}-1}\left[\tau L_{f} \sum_{n=1}^{s}\left|\delta_{t} \varepsilon_{i j}^{n-\frac{1}{2}}\right|\left|u_{i j}^{n-1}-\widetilde{u}_{i j}^{n-1}\right|\right] \\
& =L_{f} h_{1} h_{2} \sum_{i=1}^{M_{1}-1} \sum_{j=1}^{M_{2}-1}\left[\tau \sum_{n=1}^{s}\left|\delta_{t} \varepsilon_{i j}^{n-\frac{1}{2}} \| \varepsilon_{i j}^{n-1}\right|\right] \\
& \leq L_{f} h_{1} h_{2} \sum_{i=1}^{M_{1}-1} \sum_{j=1}^{M_{2}-1} \tau \sum_{n=1}^{s}\left[\frac{K_{s}}{2 L_{f}}\left(\delta_{t} \varepsilon_{i j}^{n-\frac{1}{2}}\right)^{2}+\frac{L_{f}}{2 K_{s}}\left(\varepsilon_{i j}^{n-1}\right)^{2}\right] \\
& =\frac{\tau K_{s}}{2} \sum_{n=1}^{s}\left\|\delta_{t} \varepsilon^{n-\frac{1}{2}}\right\|^{2}+\frac{\tau L_{f}^{2}}{2 K_{s}} \sum_{n=1}^{s}\left\|\varepsilon^{n-1}\right\|^{2} .
\end{aligned}
$$

From Eqs. (30)-(34), the following inequality holds:

$$
\begin{aligned}
\left\|\delta_{x} \varepsilon^{s}\right\|^{2}+\left\|\delta_{y} \varepsilon^{s}\right\|^{2} \leq & \left\|\delta_{x} \varepsilon^{0}\right\|^{2}+\left\|\delta_{y} \varepsilon^{0}\right\|^{2} \\
& +\Delta \beta \sum_{l=1}^{K} p\left(\beta_{l}\right) \frac{t_{s}^{2-\beta_{l}}}{\Gamma\left(3-\beta_{l}\right)}\left\|\psi_{2}^{*}\right\|^{2}+\frac{\tau L_{f}^{2}}{K_{s}} \sum_{n=1}^{s}\left\|\varepsilon^{n-1}\right\|^{2} .
\end{aligned}
$$

From Lemmas 1 and 2, we deduce using (35) that

$$
\begin{aligned}
\left\|\varepsilon^{n}\right\|^{2} \leq & \frac{L_{1}^{4} L_{2}^{4}}{36\left(L_{1}^{2}+L_{2}^{2}\right)^{2}}\left\|\Delta_{h} \varepsilon^{0}\right\|^{2}+\frac{L_{1}^{2} L_{2}^{2}}{6\left(L_{1}^{2}+L_{2}^{2}\right)} \Delta \beta \sum_{l=1}^{K} p\left(\beta_{l}\right) \frac{T^{2-\beta_{l}}}{\Gamma\left(3-\beta_{l}\right)}\left\|\psi_{2}^{*}\right\|^{2} \\
& +\frac{\tau L_{f}^{2} L_{1}^{2} L_{2}^{2}}{6\left(L_{1}^{2}+L_{2}^{2}\right) \Delta \beta \sum_{l=1}^{K} p\left(\beta_{l}\right) \frac{T^{1-\beta_{l}}}{\Gamma\left(2-\beta_{l}\right)}} \sum_{k=1}^{n}\left\|\varepsilon^{k-1}\right\|^{2}, \quad 1 \leq n \leq N .
\end{aligned}
$$

Finally, applying Lemma 4, we derive

$$
\begin{aligned}
\left\|\varepsilon^{n}\right\|^{2} \leq & \left(\frac{L_{1}^{4} L_{2}^{4}}{36\left(L_{1}^{2}+L_{2}^{2}\right)^{2}}\left\|\Delta_{h} \varepsilon^{0}\right\|^{2}+\frac{L_{1}^{2} L_{2}^{2}}{6\left(L_{1}^{2}+L_{2}^{2}\right)} \Delta \beta \sum_{l=1}^{K} p\left(\beta_{l}\right) \frac{T^{2-\beta_{l}}}{\Gamma\left(3-\beta_{l}\right)}\left\|\psi_{2}^{*}\right\|^{2}\right) \\
& \cdot \exp \left(\frac{L_{f}^{2} L_{1}^{2} L_{2}^{2}}{6\left(L_{1}^{2}+L_{2}^{2}\right) \Delta \beta \sum_{l=1}^{K} p\left(\beta_{l}\right) \frac{T^{-\beta_{l}}}{\Gamma\left(2-\beta_{l}\right)}}\right), \quad 1 \leq n \leq N
\end{aligned}
$$

which completes the proof. 


\subsection{Convergence}

In this part, the convergence of the difference approximation is discussed. Notice that if $U_{i j}^{n}$ is the exact solution of the system (1)-(3) and $u_{i j}^{n}$ is the numerical solution of the difference scheme (19)-(21), then the error is denoted as

$$
e_{i j}^{n}=U_{i j}^{n}-u_{i j}^{n}, \quad 0 \leq i \leq M_{1}, 0 \leq j \leq M_{2}, 0 \leq n \leq N .
$$

Subtracting (19)-(21) from (16)-(18), we get the error equations

$$
\begin{aligned}
& \Delta \beta \sum_{l=1}^{K} p\left(\beta_{l}\right) \frac{\tau^{1-\beta_{l}}}{\Gamma\left(3-\beta_{l}\right)}\left[a_{0}^{\left(\beta_{l}\right)} \delta_{t} e_{i j}^{n-\frac{1}{2}}-\sum_{k=1}^{n-1}\left(a_{n-k-1}^{\left(\beta_{l}\right)}-a_{n-k}^{\left(\beta_{l}\right)}\right) \delta_{t} e_{i j}^{k-\frac{1}{2}}\right] \\
& \quad+\frac{\tau}{4 \mu} \delta_{x}^{2} \delta_{y} \frac{e_{i j}^{n}-e_{i j}^{n-1}}{\tau} \\
&= \delta_{x}^{2} e_{i j}^{n-\frac{1}{2}}+\delta_{y}^{2} e_{i j}^{n-\frac{1}{2}}+f\left(x_{i}, y_{j}, t_{n-1}, U_{i j}^{n-1}\right)-f\left(x_{i}, y_{j}, t_{n-1}, u_{i j}^{n-1}\right) \\
&+R_{i j}^{n-\frac{1}{2}}+\widetilde{R}_{i j}^{n-\frac{1}{2}}+\widehat{R}_{i j}^{n-\frac{1}{2}}, \quad 1 \leq i \leq M_{1}-1,1 \leq j \leq M_{2}-1,1 \leq n \leq N, \\
& e_{i j}^{0}=0, \quad 1 \leq i \leq M_{1}-1,1 \leq j \leq M_{2}-1, \\
& e_{i j}^{n}=0, \quad(i, j) \in \gamma, 0 \leq n \leq N .
\end{aligned}
$$

Theorem 3 Suppose that the continuous problem (1)-(3) has a solution $u(x, y, t) \in$ $\mathcal{C}_{x, y, t}^{4,4,3}(\bar{\Omega} \times[0, T])$ and condition (4) is satisfied. Then there is a positive constant $C$ such that

$$
\left\|e^{n}\right\| \leq C\left(\tau+h_{1}^{2}+h_{2}^{2}+\Delta \beta^{2}\right) .
$$

Proof Multiplying (36) by $h_{1} h_{2} \tau \delta_{t} e_{i j}^{n-\frac{1}{2}}$, summing over $i$ from 1 to $M_{1}-1$, over $j$ from 1 to $M_{2}-1$, and over $n$ from 1 to $s$, each term in the resulted equation is estimated.

Firstly, employing an analogous strategy as that used in (30)-(34), we have the following equations:

$$
\begin{gathered}
\Delta \beta \sum_{l=1}^{K} p\left(\beta_{l}\right) \frac{\tau^{2-\beta_{l}}}{\Gamma\left(3-\beta_{l}\right)} h_{1} h_{2} \sum_{i=1}^{M_{1}-1} \sum_{j=1}^{M_{2}-1}\left\{\sum _ { n = 1 } ^ { s } \left[a_{0}^{\left(\beta_{l}\right)} \delta_{t} e_{i j}^{n-\frac{1}{2}}\right.\right. \\
\left.\left.-\sum_{k=1}^{n-1}\left(a_{n-k-1}^{\left(\beta_{l}\right)}-a_{n-k}^{\left(\beta_{l}\right)}\right) \delta_{t} e_{i j}^{k-\frac{1}{2}}\right] \delta_{t} e_{i j}^{n-\frac{1}{2}}\right\} \\
\geq \frac{1}{2} \tau K_{s} \sum_{n=1}^{s}\left\|\delta_{t} e^{n-\frac{1}{2}}\right\|^{2}, \\
h_{1} h_{2} \tau \sum_{i=1}^{M_{1}-1} \sum_{j=1}^{M_{2}-1} \sum_{n=1}^{s} \frac{\tau}{4 \mu} \delta_{x}^{2} \delta_{y} \frac{e_{i j}^{n}-e_{i j}^{n-1}}{\tau} \delta_{t} e_{i j}^{n-\frac{1}{2}} \\
=\frac{1}{4 \mu} \sum_{n=1}^{s}\left\|\delta_{x} \delta_{y}\left(e^{n}-e^{n-1}\right)\right\|^{2} \geq 0,
\end{gathered}
$$




$$
\begin{aligned}
& \tau \sum_{n=1}^{s}\left[h_{1} h_{2} \sum_{i=1}^{M_{1}-1} \sum_{j=1}^{M_{2}-1}\left(\delta_{t} e_{i j}^{n-\frac{1}{2}}\right)\left(\delta_{x}^{2} e_{i j}^{n-\frac{1}{2}}\right)\right]=-\frac{1}{2}\left\|\delta_{x} e^{s}\right\|^{2}, \\
& \tau \sum_{n=1}^{s}\left[h_{1} h_{2} \sum_{i=1}^{M_{1}-1} \sum_{j=1}^{M_{2}-1}\left(\delta_{t} e_{i j}^{n-\frac{1}{2}}\right)\left(\delta_{y}^{2} e_{i j}^{n-\frac{1}{2}}\right)\right]=-\frac{1}{2}\left\|\delta_{y} e^{s}\right\|^{2},
\end{aligned}
$$

and

$$
\begin{aligned}
& h_{1} h_{2} \sum_{i=1}^{M_{1}-1} \sum_{j=1}^{M_{2}-1}\left[\tau \sum_{n=1}^{s}\left(\delta_{t} e_{i j}^{n-\frac{1}{2}}\right)\left(f\left(x_{i}, y_{j}, t_{n-1}, U_{i j}^{n-1}\right)-f\left(x_{i}, y_{j}, t_{n-1}, u_{i j}^{n-1}\right)\right)\right] \\
& \leq h_{1} h_{2} \sum_{i=1}^{M_{1}-1} \sum_{j=1}^{M_{2}-1}\left[\tau \sum_{n=1}^{s}\left|\delta_{t} e_{i j}^{n-\frac{1}{2}}\right|\left|f\left(x_{i}, y_{j}, t_{n-1}, U_{i j}^{n-1}\right)-f\left(x_{i}, y_{j}, t_{n-1}, u_{i j}^{n-1}\right)\right|\right] \\
& \leq L_{f} h_{1} h_{2} \sum_{i=1}^{M_{1}-1} \sum_{j=1}^{M_{2}-1}\left[\tau \sum_{n=1}^{s}\left|\delta_{t} e_{i j}^{n-\frac{1}{2}}\right|\left|e_{i j}^{n-1}\right|\right] \\
& \leq L_{f} h_{1} h_{2} \sum_{i=1}^{M_{1}-1} \sum_{j=1}^{M_{2}-1} \tau \sum_{n=1}^{s}\left[\frac{K_{s}}{4 L_{f}}\left(\delta_{t} e_{i j}^{n-\frac{1}{2}}\right)^{2}+\frac{L_{f}}{K_{s}}\left(e_{i j}^{n-1}\right)^{2}\right] \\
& =\frac{\tau K_{s}}{4} \sum_{n=1}^{s}\left\|\delta_{t} e^{n-\frac{1}{2}}\right\|^{2}+\frac{\tau L_{f}^{2}}{K_{s}} \sum_{n=1}^{s}\left\|e^{n-1}\right\|^{2} .
\end{aligned}
$$

Then for the term containing the remainders, it can be deduced that

$$
\begin{aligned}
h_{1} h_{2} & \sum_{i=1}^{M_{1}-1} \sum_{j=1}^{M_{2}-1} \sum_{n=1}^{s} \tau\left(\delta_{t} e_{i j}^{n-\frac{1}{2}}\right)\left(R_{i j}^{n-\frac{1}{2}}+\widetilde{R}_{i j}^{n-\frac{1}{2}}+\widehat{R}_{i j}^{n-\frac{1}{2}}\right) \\
\leq & h_{1} h_{2} \sum_{i=1}^{M_{1}-1} \sum_{j=1}^{M_{2}-1} \sum_{n=1}^{s} \tau\left(\frac{K_{s}}{4}\left(\delta_{t} e_{i j}^{n-\frac{1}{2}}\right)^{2}+\frac{1}{K_{s}}\left(R_{i j}^{n-\frac{1}{2}}+\widetilde{R}_{i j}^{n-\frac{1}{2}}+\widehat{R}_{i j}^{n-\frac{1}{2}}\right)^{2}\right) \\
\leq & \frac{\tau K_{s}}{4} \sum_{n=1}^{s}\left\|\delta_{t} e^{n-\frac{1}{2}}\right\|^{2}+\frac{\tau h_{1} h_{2}}{K_{s}} \sum_{i=1}^{M_{1}-1} \sum_{j=1}^{M_{2}-1} \sum_{n=1}^{s}\left[C_{3}\left(\tau^{1+\frac{\Delta \beta}{2}}+h_{1}^{2}+h_{2}^{2}+\Delta \beta^{2}\right)\right. \\
& \left.+C_{4} \tau+C_{5} \tau^{3}|\ln \tau|\right]^{2} \\
\leq & \frac{\tau K_{s}}{4} \sum_{n=1}^{s}\left\|\delta_{t} e^{n-\frac{1}{2}}\right\|^{2}+\frac{\tau h_{1} h_{2}}{K_{s}} \sum_{i=1}^{M_{1}-1} \sum_{j=1}^{M_{2}-1} \sum_{n=1}^{s}\left[\left(C_{3}+C_{4}+C_{5}\right)\left(\tau+h_{1}^{2}+h_{2}^{2}+\Delta \beta^{2}\right)\right]^{2} \\
\leq & \frac{\tau K_{s}}{4} \sum_{n=1}^{s}\left\|\delta_{t} e^{n-\frac{1}{2}}\right\|^{2}+\frac{T L_{1} L_{2}}{K_{s}}\left[\left(C_{3}+C_{4}+C_{5}\right)\left(\tau+h_{1}^{2}+h_{2}^{2}+\Delta \beta^{2}\right)\right]^{2} .
\end{aligned}
$$

Due to (37)-(42), this yields

$$
\begin{aligned}
\frac{1}{2}\left(\left\|\delta_{x} e^{s}\right\|^{2}+\left\|\delta_{y} e^{s}\right\|^{2}\right) \leq & \frac{T L_{1} L_{2}}{K_{s}}\left[\left(C_{3}+C_{4}+C_{5}\right)\left(\tau+h_{1}^{2}+h_{2}^{2}+\Delta \beta^{2}\right)\right]^{2} \\
& +\frac{\tau L_{f}^{2}}{K_{s}} \sum_{n=1}^{s}\left\|e^{n-1}\right\|^{2}
\end{aligned}
$$


i.e.,

$$
\begin{aligned}
\frac{1}{2}\left|e^{s}\right|_{1}^{2} \leq & \frac{T L_{1} L_{2}}{\Delta \beta \sum_{l=1}^{K} p\left(\beta_{l}\right) \frac{1}{\Gamma\left(2-\beta_{l}\right)} T^{1-\beta_{l}}}\left[\left(C_{3}+C_{4}+C_{5}\right)\left(\tau+h_{1}^{2}+h_{2}^{2}+\Delta \beta^{2}\right)\right]^{2} \\
& +\frac{\tau L_{f}^{2}}{K_{s}} \sum_{n=1}^{s}\left\|e^{n-1}\right\|^{2} .
\end{aligned}
$$

From Lemma 1, we obtain

$$
\begin{aligned}
\left\|e^{n}\right\|^{2} \leq & \frac{L_{1}^{3} L_{2}^{3}}{3\left(L_{1}^{2}+L_{2}^{2}\right) \Delta \beta \sum_{l=1}^{K} p\left(\beta_{l}\right) \frac{T^{-}-\beta_{l}}{\Gamma\left(2-\beta_{l}\right)}}\left[\left(C_{3}+C_{4}+C_{5}\right)\left(\tau+h_{1}^{2}+h_{2}^{2}+\Delta \beta^{2}\right)\right]^{2} \\
& +\frac{\tau L_{f}^{2} L_{1}^{2} L_{2}^{2}}{3\left(L_{1}^{2}+L_{2}^{2}\right) \Delta \beta \sum_{l=1}^{K} p\left(\beta_{l}\right) \frac{T^{1-\beta_{l}}}{\Gamma\left(2-\beta_{l}\right)}} \sum_{k=1}^{n}\left\|e^{k-1}\right\|^{2}, \quad 1 \leq n \leq N .
\end{aligned}
$$

Therefore, by using Lemma 4, we have

$$
\begin{aligned}
\left\|e^{n}\right\|^{2} \leq & \frac{L_{1}^{3} L_{2}^{3}}{3\left(L_{1}^{2}+L_{2}^{2}\right) \Delta \beta \sum_{l=1}^{K} p\left(\beta_{l}\right) \frac{T^{-} \beta_{l}}{\Gamma\left(2-\beta_{l}\right)}}\left[\left(C_{3}+C_{4}+C_{5}\right)\left(\tau+h_{1}^{2}+h_{2}^{2}+\Delta \beta^{2}\right)\right]^{2} \\
& \cdot \exp \left(\frac{L_{f}^{2} L_{1}^{2} L_{2}^{2}}{3\left(L_{1}^{2}+L_{2}^{2}\right) \Delta \beta \sum_{l=1}^{K} p\left(\beta_{l}\right) \frac{T^{-\beta_{l}}}{\Gamma\left(2-\beta_{l}\right)}}\right), \quad 1 \leq n \leq N .
\end{aligned}
$$

This completes the proof.

\section{Description of the ADI scheme}

For ease of computation, the ADI scheme is developed in this section. Considering Eqs. (19)-(21), and noticing $a_{0}^{\left(\beta_{l}\right)}=1$, we rewrite Eq. (19) as

$$
\begin{aligned}
& \Delta \beta \sum_{l=1}^{K} p\left(\beta_{l}\right) \frac{1}{\tau^{\beta_{l}} \Gamma\left(3-\beta_{l}\right)} u_{i j}^{n}-\frac{1}{2} \delta_{x}^{2} u_{i j}^{n}-\frac{1}{2} \delta_{y}^{2} u_{i j}^{n}+\frac{1}{4 \mu} \delta_{x}^{2} \delta_{y}^{2} u_{i j}^{n} \\
& =\Delta \beta \sum_{l=1}^{K} p\left(\beta_{l}\right) \frac{1}{\tau^{\beta_{l}} \Gamma\left(3-\beta_{l}\right)}\left[u_{i j}^{n-1}+\sum_{k=1}^{n-1}\left(a_{n-k-1}^{\left(\beta_{l}\right)}-a_{n-k}^{\left(\beta_{l}\right)}\right)\left(u_{i j}^{k}-u_{i j}^{k-1}\right)\right. \\
& \left.\quad+\tau a_{n-1}^{\left(\beta_{l}\right)}\left(\psi_{2}\right)_{i j}\right]+\frac{1}{2} \delta_{x}^{2} u_{i j}^{n-1}+\frac{1}{2} \delta_{y}^{2} u_{i j}^{n-1}+\frac{1}{4 \mu} \delta_{x}^{2} \delta_{y}^{2} u_{i j}^{n-1}+f\left(x_{i}, y_{j}, t_{n-1}, u_{i j}^{n-1}\right),
\end{aligned}
$$

or

$$
\begin{aligned}
( & \left.\sqrt{\mu} I-\frac{1}{2 \sqrt{\mu}} \delta_{x}^{2}\right)\left(\sqrt{\mu} I-\frac{1}{2 \sqrt{\mu}} \delta_{y}^{2}\right) u_{i j}^{n} \\
= & \left(\sqrt{\mu} I+\frac{1}{2 \sqrt{\mu}} \delta_{x}^{2}\right)\left(\sqrt{\mu} I+\frac{1}{2 \sqrt{\mu}} \delta_{y}^{2}\right) u_{i j}^{n-1}+\Delta \beta \sum_{l=1}^{K} p\left(\beta_{l}\right) \frac{1}{\tau^{\beta_{l}} \Gamma\left(3-\beta_{l}\right)} \\
& \cdot\left[\sum_{k=1}^{n-1}\left(a_{n-k-1}^{\left(\beta_{l}\right)}-a_{n-k}^{\left(\beta_{l}\right)}\right)\left(u_{i j}^{k}-u_{i j}^{k-1}\right)+\tau a_{n-1}^{\left(\beta_{l}\right)}\left(\psi_{2}\right)_{i j}\right]+f\left(x_{i}, y_{j}, t_{n-1}, u_{i j}^{n-1}\right),
\end{aligned}
$$

where $I$ denotes the identity operator. 
Let

$$
u_{i j}^{*}=\left(\sqrt{\mu} I-\frac{1}{2 \sqrt{\mu}} \delta_{y}^{2}\right) u_{i j}^{n}
$$

Then we have the ADI form of difference scheme (19)-(21), and the procedure can be executed as follows:

At each time instance $t=t_{n}(1 \leq n \leq N)$, firstly, for all fixed $y=y_{j}\left(1 \leq j \leq M_{2}-1\right)$, by solving a set of $M_{1}-1$ equations at the mesh points $x_{i}\left(1 \leq i \leq M_{1}-1\right)$, we get the intermediate solution $u_{i j}^{*}$ :

$$
\left\{\begin{aligned}
&\left(\sqrt{\mu} I-\frac{1}{2 \sqrt{\mu}} \delta_{x}^{2}\right) u_{i j}^{*} \\
&=\left(\sqrt{\mu} I+\frac{1}{2 \sqrt{\mu}} \delta_{x}^{2}\right)\left(\sqrt{\mu} I+\frac{1}{2 \sqrt{\mu}} \delta_{y}^{2}\right) u_{i j}^{n-1} \\
&+\Delta \beta \sum_{l=1}^{K} p\left(\beta_{l}\right) \frac{1}{\tau^{\beta} l \Gamma\left(3-\beta_{l}\right)}\left[\sum_{k=1}^{n-1}\left(a_{n-k-1}^{\left(\beta_{l}\right)}-a_{n-k}^{\left(\beta_{l}\right)}\right)\left(u_{i j}^{k}-u_{i j}^{k-1}\right)\right. \\
&\left.+\tau a_{n-1}^{\left(\beta_{l}\right)}\left(\psi_{2}\right)_{i j}\right]+f\left(x_{i}, y_{j}, t_{n-1}, u_{i j}^{n-1}\right), \quad 1 \leq i \leq M_{1}-1, \\
& u_{0 j}^{*}=\left(\sqrt{\mu} I-\frac{1}{2 \sqrt{\mu}} \delta_{y}^{2}\right) u_{0 j}^{n}, \quad u_{M_{1} j}^{*}=\left(\sqrt{\mu} I-\frac{1}{2 \sqrt{\mu}} \delta_{y}^{2}\right) u_{M_{1 j}}^{n} ;
\end{aligned}\right.
$$

afterwards, for all fixed $x=x_{i}\left(1 \leq i \leq M_{1}-1\right)$, by solving a set of $M_{2}-1$ equations at the mesh points $y_{j}\left(1 \leq j \leq M_{2}-1\right)$, the solution $u_{i j}^{n}$ can be obtained:

$$
\left\{\begin{array}{l}
\left(\sqrt{\mu} I-\frac{1}{2 \sqrt{\mu}} \delta_{y}^{2}\right) u_{i j}^{n}=u_{i j}^{*}, \quad 1 \leq j \leq M_{2}-1, \\
u_{i 0}^{n}=\phi\left(x_{i}, y_{0}, t_{n}\right), \quad u_{i M_{2}}^{n}=\phi\left(x_{i}, y_{M_{2}}, t_{n}\right) .
\end{array}\right.
$$

Also, the ADI form of (22)-(24) can be described as:

At each time instance $t=t_{n}(1 \leq n \leq N)$, firstly, for all fixed $y=y_{j}\left(1 \leq j \leq M_{2}-1\right)$, solving a set of $M_{1}-1$ equations at the mesh points $x_{i}\left(1 \leq i \leq M_{1}-1\right)$, the intermediate solution $u_{i j}^{*}$ is derived:

$$
\left\{\begin{aligned}
(\sqrt{\tilde{\mu}} I & \left.-\frac{1}{2 \sqrt{\mu}} \delta_{x}^{2}\right) u_{i j}^{*} \\
= & \left(\sqrt{\mu} I+\frac{1}{2 \sqrt{\mu}} \delta_{x}^{2}\right)\left(\sqrt{\mu} I+\frac{1}{2 \sqrt{\mu}} \delta_{y}^{2}\right) u_{i j}^{n-1} \\
& +\frac{\Delta \beta}{2} \sum_{l=1}^{K} p\left(\widetilde{\beta}_{l}^{(1)}\right) \frac{1}{\tau^{(1)} \Gamma\left(3-\widetilde{\beta}_{l}^{(1)}\right)}\left[\sum_{k=1}^{n-1}\left(a_{n-k-1}^{\left(\widetilde{\beta}_{l}^{(1)}\right)}-a_{n-k}^{\left(\widetilde{\beta}_{l}^{(1)}\right)}\right)\left(u_{i j}^{k}-u_{i j}^{k-1}\right)\right. \\
& \left.+\tau a_{n-1}^{\left(\widetilde{\beta}_{l}^{(1)}\right)}\left(\psi_{2}\right)_{i j}\right] \\
& +\frac{\Delta \beta}{2} \sum_{l=1}^{K} p\left(\widetilde{\beta}_{l}^{(2)}\right) \frac{1}{\tau^{(2)} \widetilde{\beta}_{l}^{(2)} \Gamma\left(3-\widetilde{\beta}_{l}^{(2)}\right)}\left[\sum_{k=1}^{n-1}\left(a_{n-k-1}^{\left(\widetilde{\beta}_{l}^{(2)}\right)}-a_{n-k}^{\left(\widetilde{\beta}_{l}^{(2)}\right)}\right)\left(u_{i j}^{k}-u_{i j}^{k-1}\right)\right. \\
& \left.+\tau a_{n-1}^{\left(\widetilde{\beta}_{l}^{(2)}\right)}\left(\psi_{2}\right)_{i j}\right]+f\left(x_{i}, y_{j}, t_{n-1}, u_{i j}^{n-1}\right), \quad 1 \leq i \leq M_{1}-1, \\
u_{0 j}^{*}= & \left(\sqrt{\tilde{\mu}} I-\frac{1}{2 \sqrt{\mu}} \delta_{y}^{2}\right) u_{0 j}^{n}, \quad u_{M_{1} j}^{*}=\left(\sqrt{\tilde{\mu}} I-\frac{1}{2 \sqrt{\mu}} \delta_{y}^{2}\right) u_{M_{1} j}^{n} ;
\end{aligned}\right.
$$

next, for all fixed $x=x_{i}\left(1 \leq i \leq M_{1}-1\right)$, by solving a set of $M_{2}-1$ equations at the mesh points $y_{j}\left(1 \leq j \leq M_{2}-1\right)$, the solution $u_{i j}^{n}$ can be obtained:

$$
\left\{\begin{array}{l}
\left(\sqrt{\widetilde{\mu}} I-\frac{1}{2 \sqrt{\mu}} \delta_{y}^{2}\right) u_{i j}^{n}=u_{i j}^{*}, \quad 1 \leq j \leq M_{2}-1, \\
u_{i 0}^{n}=\phi\left(x_{i}, y_{0}, t_{n}\right), \quad u_{i M_{2}}^{n}=\phi\left(x_{i}, y_{M_{2}}, t_{n}\right) .
\end{array}\right.
$$




\section{Three-dimensional problem}

In what follows, we present numerical schemes for the three-dimensional problem.

Consider the three-dimensional time-fractional wave equation of distributed-order with a nonlinear source term along with its initial and boundary conditions:

$$
\begin{aligned}
& \int_{1}^{2} p(\beta)_{0}^{C} D_{t}^{\beta} u(x, y, z, t) d \beta \\
& =\frac{\partial^{2} u(x, y, z, t)}{\partial x^{2}}+\frac{\partial^{2} u(x, y, z, t)}{\partial y^{2}}+\frac{\partial^{2} u(x, y, z, t)}{\partial z^{2}} \\
& \quad+f(x, y, z, t, u(x, y, z, t)), \quad(x, y, z) \in \Omega, t \in(0, T], \\
& u(x, y, z, t)=\phi(x, y, z, t), \quad(x, y, z) \in \partial \Omega, t \in[0, T], \\
& u(x, y, z, 0)=\psi_{1}(x, y, z), \quad u_{t}(x, y, z, 0)=\psi_{2}(x, y, z), \quad(x, y, z) \in \Omega,
\end{aligned}
$$

where $\Omega=\left(0, L_{1}\right) \times\left(0, L_{2}\right) \times\left(0, L_{3}\right)$, and $\partial \Omega$ is the boundary of $\Omega$. We still assume that $p(\beta), \phi(x, y, z, t), \psi_{1}(x, y, z), \psi_{2}(x, y, z)$ and $f(x, y, z, t, u)$ are continuous, and the nonlinear source term $f$ satisfies a Lipschitz condition of the form

$$
\left|f\left(x, y, z, t, u_{1}\right)-f\left(x, y, z, t, u_{2}\right)\right| \leq L_{f}\left|u_{1}-u_{2}\right|
$$

where $L_{f}$ is a positive constant.

Let $M_{1}, M_{2}, M_{3}$ and $N$ be positive integers, and $h_{i}=L_{i} / M_{i}(i=1,2,3)$ and $\tau=T / N$ represent the uniform step sizes in spatial and temporal directions, respectively. Denote

$$
\begin{aligned}
& \Omega_{h}=\left\{\left(x_{i}, y_{j}, z_{m}\right) \mid 1 \leq i \leq M_{1}-1,1 \leq j \leq M_{2}-1,1 \leq m \leq M_{3}-1\right\}, \\
& \partial \Omega_{h}=\left\{\left(x_{i}, y_{j}, z_{m}\right) \mid i=0 \text { or } i=M_{1} \text { or } j=0 \text { or } j=M_{2} \text { or } m=0 \text { or } m=M_{3}\right\}, \\
& \bar{\Omega}_{h}=\Omega_{h} \cup \partial \Omega_{h}, \\
& \Omega_{\tau}=\left\{t_{n} \mid t_{n}=n \tau, 0 \leq n \leq N\right\} .
\end{aligned}
$$

Then the partition of $\bar{\Omega} \times[0, T]$ is $\bar{\Omega}_{h} \times \Omega_{\tau}$.

Take $U_{i j m}^{n}=u\left(x_{i}, y_{j}, z_{m}, t_{n}\right)$, the grid functions on $\bar{\Omega}_{h} \times \Omega_{\tau}$, with $0 \leq i \leq M_{1}, 0 \leq j \leq M_{2}$, $0 \leq m \leq M_{3}, 0 \leq n \leq N$. Similar to the derivation of Eq. (14), we get the equation

$$
\begin{aligned}
\Delta \beta & \sum_{l=1}^{K} p\left(\beta_{l}\right) \frac{\tau^{1-\beta_{l}}}{\Gamma\left(3-\beta_{l}\right)}\left[a_{0}^{\left(\beta_{l}\right)} \delta_{t} U_{i j m}^{n-\frac{1}{2}}-\sum_{k=1}^{n-1}\left(a_{n-k-1}^{\left(\beta_{l}\right)}-a_{n-k}^{\left(\beta_{l}\right)}\right) \delta_{t} U_{i j m}^{k-\frac{1}{2}}\right. \\
& \left.-a_{n-1}^{\left(\beta_{l}\right)} \psi_{2}\left(x_{i}, y_{j}, z_{m}\right)\right] \\
= & \delta_{x}^{2} U_{i j m}^{n-\frac{1}{2}}+\delta_{y}^{2} U_{i j m}^{n-\frac{1}{2}}+\delta_{z}^{2} U_{i j m}^{n-\frac{1}{2}}+f\left(x_{i}, y_{j}, z_{m}, t_{n-1}, U_{i j m}^{n-1}\right)+R_{i j m}^{n-\frac{1}{2}}+\widetilde{R}_{i j m}^{n-\frac{1}{2}},
\end{aligned}
$$

where there exist positive constants $C_{6}$ and $C_{7}$ such that

$$
\left|R_{i j m}^{n-\frac{1}{2}}\right| \leq C_{6}\left(\tau^{1+\frac{1}{2} \Delta \beta}+h_{1}^{2}+h_{2}^{2}+h_{3}^{2}+\Delta \beta^{2}\right)
$$


and

$$
\left|\widetilde{R}_{i j m}^{n-\frac{1}{2}}\right| \leq C_{7} \tau
$$

Adding the high order term

$$
\begin{aligned}
\widehat{R}_{i j m}^{n-\frac{1}{2}}= & \frac{\tau}{4 \mu} \delta_{x}^{2} \delta_{y}^{2} \frac{U_{i j m}^{n}-U_{i j m}^{n-1}}{\tau}+\frac{\tau}{4 \mu} \delta_{y}^{2} \delta_{z}^{2} \frac{U_{i j m}^{n}-U_{i j m}^{n-1}}{\tau}+\frac{\tau}{4 \mu} \delta_{z}^{2} \delta_{x}^{2} \frac{U_{i j m}^{n}-U_{i j m}^{n-1}}{\tau} \\
& -\frac{1}{8 \mu^{2}} \delta_{x}^{2} \delta_{y}^{2} \delta_{z}^{2}\left(U_{i j m}^{n}+U_{i j m}^{n-1}\right)
\end{aligned}
$$

on both sides of (51), we derive

$$
\begin{aligned}
& \Delta \beta \sum_{l=1}^{K} p\left(\beta_{l}\right) \frac{\tau^{1-\beta_{l}}}{\Gamma\left(3-\beta_{l}\right)}\left[a_{0}^{\left(\beta_{l}\right)} \delta_{t} U_{i j m}^{n-\frac{1}{2}}-\sum_{k=1}^{n-1}\left(a_{n-k-1}^{\left(\beta_{l}\right)}-a_{n-k}^{\left(\beta_{l}\right)}\right) \delta_{t} U_{i j m}^{k-\frac{1}{2}}\right. \\
& \left.\quad-a_{n-1}^{\left(\beta_{l}\right)} \psi_{2}\left(x_{i}, y_{j}, z_{m}\right)\right]+\frac{\tau}{4 \mu} \delta_{x}^{2} \delta_{y}^{2} \frac{U_{i j m}^{n}-U_{i j m}^{n-1}}{\tau}+\frac{\tau}{4 \mu} \delta_{y}^{2} \delta_{z}^{2} \frac{U_{i j m}^{n}-U_{i j m}^{n-1}}{\tau} \\
& \quad+\frac{\tau}{4 \mu} \delta_{z}^{2} \delta_{x}^{2} \frac{U_{i j m}^{n}-U_{i j m}^{n-1}}{\tau}-\frac{1}{8 \mu^{2}} \delta_{x}^{2} \delta_{y}^{2} \delta_{z}^{2}\left(U_{i j m}^{n}+U_{i j m}^{n-1}\right) \\
& =\delta_{x}^{2} U_{i j m}^{n-\frac{1}{2}}+\delta_{y}^{2} U_{i j m}^{n-\frac{1}{2}}+\delta_{z}^{2} U_{i j m}^{n-\frac{1}{2}}+f\left(x_{i}, y_{j}, z_{m}, t_{n-1}, U_{i j m}^{n-1}\right)+R_{i j m}^{n-\frac{1}{2}}+\widetilde{R}_{i j m}^{n-\frac{1}{2}} \\
& \quad+\widehat{R}_{i j m}^{n-\frac{1}{2}} .
\end{aligned}
$$

From Eq. (52), it is observed that

$$
\left|\widehat{R}_{i j m}^{n-\frac{1}{2}}\right| \leq C_{8} \tau^{3}|\ln \tau|
$$

where $C_{8}$ is a positive constant. For the initial and boundary conditions, we have

$$
\begin{aligned}
& U_{i j m}^{0}=\psi_{1}\left(x_{i}, y_{j}, z_{m}\right), \quad\left(x_{i}, y_{j}, z_{m}\right) \in \Omega_{h}, \\
& U_{i j m}^{n}=\phi\left(x_{i}, y_{j}, z_{m}, t_{n}\right), \quad\left(x_{i}, y_{j}, z_{m}\right) \in \partial \Omega_{h}, 0 \leq n \leq N .
\end{aligned}
$$

Let $u_{i j m}^{n}$ be a numerical approximation to $u\left(x_{i}, y_{j}, z_{m}, t_{n}\right)$. Neglecting the small terms $R_{i j m}^{n-\frac{1}{2}}$, $\widetilde{R}_{i j m}^{n-\frac{1}{2}}$ and $\widehat{R}_{i j m}^{n-\frac{1}{2}}$ in (53), and using $u_{i j m}^{n}$ instead of $U_{i j m}^{n}$ in (53)-(55), we develop the finite difference scheme for (47)-(49) as follows:

$$
\begin{gathered}
\Delta \beta \sum_{l=1}^{K} p\left(\beta_{l}\right) \frac{\tau^{1-\beta_{l}}}{\Gamma\left(3-\beta_{l}\right)}\left[a_{0}^{\left(\beta_{l}\right)} \delta_{t} u_{i j m}^{n-\frac{1}{2}}-\sum_{k=1}^{n-1}\left(a_{n-k-1}^{\left(\beta_{l}\right)}-a_{n-k}^{\left(\beta_{l}\right)}\right) \delta_{t} u_{i j m}^{k-\frac{1}{2}}\right. \\
\left.-a_{n-1}^{\left(\beta_{l}\right)}\left(\psi_{2}\right)_{i j m}\right]+\frac{\tau}{4 \mu} \delta_{x}^{2} \delta_{y}^{2} \frac{u_{i j m}^{n}-u_{i j m}^{n-1}}{\tau}+\frac{\tau}{4 \mu} \delta_{y}^{2} \delta_{z}^{2} \frac{u_{i j m}^{n}-u_{i j m}^{n-1}}{\tau} \\
\quad+\frac{\tau}{4 \mu} \delta_{z}^{2} \delta_{x}^{2} \frac{u_{i j m}^{n}-u_{i j m}^{n-1}}{\tau}-\frac{1}{8 \mu^{2}} \delta_{x}^{2} \delta_{y}^{2} \delta_{z}^{2}\left(u_{i j m}^{n}+u_{i j m}^{n-1}\right) \\
=\delta_{x}^{2} u_{i j m}^{n-\frac{1}{2}}+\delta_{y}^{2} u_{i j m}^{n-\frac{1}{2}}+\delta_{z}^{2} u_{i j m}^{n-\frac{1}{2}}+f\left(x_{i}, y_{j}, z_{m}, t_{n-1}, u_{i j m}^{n-1}\right)
\end{gathered}
$$




$$
\begin{gathered}
1 \leq i \leq M_{1}-1,1 \leq j \leq M_{2}-1,1 \leq m \leq M_{3}-1,1 \leq n \leq N, \\
u_{i j m}^{0}=\left(\psi_{1}\right)_{i j m}, \quad 1 \leq i \leq M_{1}-1,1 \leq j \leq M_{2}-1,1 \leq m \leq M_{3}-1, \\
u_{i j m}^{n}=\phi_{i j m}^{n}, \quad(i, j, m) \in \gamma=\left\{(i, j, m) \mid\left(x_{i}, y_{j}, z_{m}\right) \in \partial \Omega_{h}\right\}, 0 \leq n \leq N,
\end{gathered}
$$

where

$$
\begin{array}{r}
\left(\psi_{1}\right)_{i j m}=\psi_{1}\left(x_{i}, y_{j}, z_{m}\right), \quad\left(\psi_{2}\right)_{i j m}=\psi_{2}\left(x_{i}, y_{j}, z_{m}\right), \\
1 \leq i \leq M_{1}-1,1 \leq j \leq M_{2}-1,1 \leq m \leq M_{3}-1
\end{array}
$$

and

$$
\phi_{i j m}^{n}=\phi\left(x_{i}, y_{j}, z_{m}, t_{n}\right), \quad(i, j, m) \in \gamma, 0 \leq n \leq N
$$

By defining the discrete norms and Sobolev seminorm corresponding to the threedimensional problems, we can prove that Lemmas 1 and 2 are available for the cuboidal domain. The theoretical analysis for the three-dimensional problem can be performed in a similar manner as of the two-dimensional case in Sect. 3, though it is much more complicated. For the sake of briefness, we won't repeat it and list the theoretical results directly. Hence, as generalizations of Theorems 1, 2 and 3, we have the following conclusions.

Theorem 4 The finite difference scheme (56)-(58) is uniquely solvable.

Theorem 5 If condition (50) is satisfied, then the difference scheme (56)-(58) is unconditionally stable.

Theorem 6 Suppose that the continuous problem (47)-(49) has a solution $u(x, y, z, t) \in$ $\mathcal{C}_{x, y, z, t}^{4,4,4,3}(\bar{\Omega} \times[0, T])$ and condition (50) is satisfied. Then there is a positive constant $C$ such that

$$
\left\|e^{n}\right\| \leq C\left(\tau+h_{1}^{2}+h_{2}^{2}+h_{3}^{2}+\Delta \beta^{2}\right) .
$$

For ease of implementation, we adopt the following ADI scheme when computing.

At each time instance $t=t_{n}(1 \leq n \leq N)$, firstly, for all fixed $y=y_{j}\left(1 \leq j \leq M_{2}-1\right)$ and $z=z_{m}\left(1 \leq m \leq M_{3}-1\right)$, solving a set of $M_{1}-1$ equations at the mesh points $x_{i}$ $\left(1 \leq i \leq M_{1}-1\right)$, the first intermediate solution $u_{i j m}^{* *}$ is given as:

$$
\left\{\begin{aligned}
(\sqrt[3]{\mu} I & \left.-\frac{1}{2 \mu^{\frac{2}{3}}} \delta_{x}^{2}\right) u_{i j m}^{* *} \\
= & \left(\sqrt[3]{\mu} I+\frac{1}{2 \mu^{\frac{2}{3}}} \delta_{x}^{2}\right)\left(\sqrt[3]{\mu} I+\frac{1}{2 \mu^{\frac{2}{3}}} \delta_{y}^{2}\right)\left(\sqrt[3]{\mu} I+\frac{1}{2 \mu^{\frac{2}{3}}} \delta_{z}^{2}\right) u_{i j m}^{n-1} \\
& +\Delta \beta \sum_{l=1}^{K} p\left(\beta_{l}\right) \frac{1}{\tau^{\beta_{l}} \Gamma\left(3-\beta_{l}\right)}\left[\sum_{k=1}^{n-1}\left(a_{n-k-1}^{\left(\beta_{l}\right)}-a_{n-k}^{\left(\beta_{l}\right)}\right)\left(u_{i j m}^{k}-u_{i j m}^{k-1}\right)\right. \\
& \left.+\tau a_{n-1}^{\left(\beta_{l}\right)}\left(\psi_{2}\right)_{i j m}\right] \\
& +f\left(x_{i}, y_{j}, z_{m}, t_{n-1}, u_{i j m}^{n-1}\right), \quad 1 \leq i \leq M_{1}-1, \\
u_{0 j m}^{* *} & \left(\sqrt[3]{\mu} I-\frac{1}{2 \mu^{\frac{2}{3}}} \delta_{y}^{2}\right) u_{0 j m}^{*}, \quad u_{M_{1} j m}^{* *}=\left(\sqrt[3]{\mu} I-\frac{1}{2 \mu^{\frac{2}{3}}} \delta_{y}^{2}\right) u_{M_{1 j}}^{*} ;
\end{aligned}\right.
$$


afterwards, for all fixed $z=z_{m}\left(1 \leq m \leq M_{3}-1\right)$ and $x=x_{i}\left(1 \leq i \leq M_{1}-1\right)$, by solving a set of $M_{2}-1$ equations at the mesh points $y_{j}\left(1 \leq j \leq M_{2}-1\right)$, the second intermediate solution $u_{i j m}^{*}$ can be obtained:

$$
\left\{\begin{array}{l}
\left(\sqrt[3]{\mu} I-\frac{1}{2 \mu^{\frac{2}{3}}} \delta_{y}^{2}\right) u_{i j m}^{*}=u_{i j m}^{* *}, \quad 1 \leq j \leq M_{2}-1 \\
u_{i 0 m}^{*}=\left(\sqrt[3]{\mu} I-\frac{1}{2 \mu^{\frac{2}{3}}} \delta_{z}^{2}\right) u_{i 0 m}^{n}, \quad u_{i M_{2} m}^{*}=\left(\sqrt[3]{\mu} I-\frac{1}{2 \mu^{\frac{2}{3}}} \delta_{z}^{2}\right) u_{i M_{2} m}^{n}
\end{array}\right.
$$

finally, for all fixed $x=x_{i}\left(1 \leq i \leq M_{1}-1\right)$ and $y=y_{j}\left(1 \leq j \leq M_{2}-1\right)$, solving a set of $M_{3}-1$ equations at the mesh points $z_{m}\left(1 \leq m \leq M_{3}-1\right)$, we have $u_{i j m}^{n}$ :

$$
\left\{\begin{array}{l}
\left(\sqrt[3]{\mu} I-\frac{1}{2 \mu^{\frac{2}{3}}} \delta_{z}^{2}\right) u_{i j m}^{n}=u_{i j m}^{*}, \quad 1 \leq m \leq M_{3}-1 \\
u_{i j 0}^{n}=\phi\left(x_{i}, y_{j}, z_{0}, t_{n}\right), \quad u_{i j M_{3}}^{n}=\phi\left(x_{i}, y_{j}, z_{M_{3}}, t_{n}\right)
\end{array}\right.
$$

Also, by employing the composite two-point Gauss-Legendre quadrature rule, the ADI scheme for the three-dimensional problem is obtained, and it is described as following: At each time instance $t=t_{n}(1 \leq n \leq N)$, firstly, for all fixed $y=y_{j}\left(1 \leq j \leq M_{2}-1\right)$ and $z=z_{m}$ (1 $\left.\leq m \leq M_{3}-1\right)$, solving a set of $M_{1}-1$ equations at the mesh points $x_{i}$ $\left(1 \leq i \leq M_{1}-1\right)$, the first intermediate solution $u_{i j m}^{* *}$ is given as:

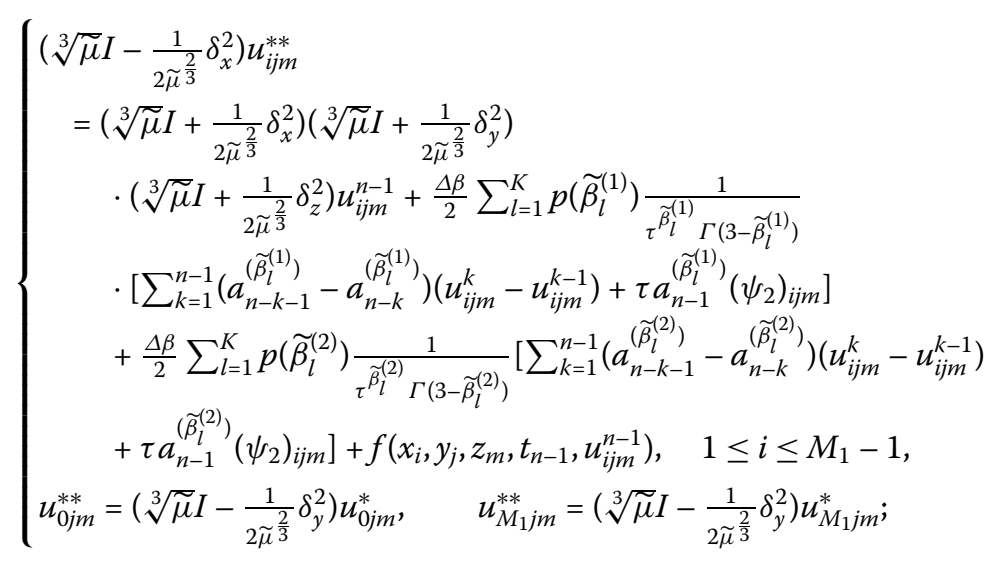

afterwards, for all fixed $z=z_{m}\left(1 \leq m \leq M_{3}-1\right)$ and $x=x_{i}\left(1 \leq i \leq M_{1}-1\right)$, by solving a set of $M_{2}-1$ equations at the mesh points $y_{j}\left(1 \leq j \leq M_{2}-1\right)$, the second intermediate solution $u_{i j m}^{*}$ can be obtained:

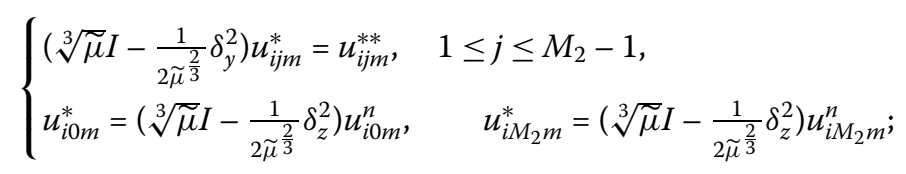

finally, for all fixed $x=x_{i}\left(1 \leq i \leq M_{1}-1\right)$ and $y=y_{j}\left(1 \leq j \leq M_{2}-1\right)$, solving a set of $M_{3}-1$ equations at the mesh points $z_{m}\left(1 \leq m \leq M_{3}-1\right)$, we have $u_{i j m}^{n}$ :

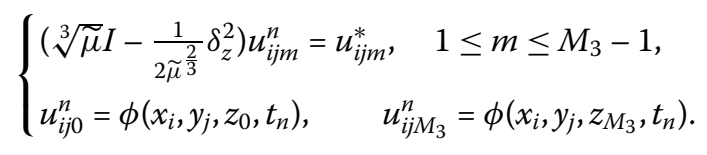




\section{Numerical results}

In this section, numerical examples are given to demonstrate the availability of the proposed schemes and verify the theoretical results, including convergence orders and numerical stability. Both the discrete $L^{2}$ and $L^{\infty}$ norms are taken to measure the numerical errors. Denote

$$
\left\|e^{N}\right\|_{L^{2}}:=\left(\sum_{m=1}^{M_{3}-1} \sum_{j=1}^{M_{2}-1} \sum_{i=1}^{M_{1}-1}\left|U_{i j m}^{N}-u_{i j m}^{N}\right|^{2} h_{1} h_{2} h_{3}\right)^{\frac{1}{2}}
$$

and

$$
\left\|e^{N}\right\|_{L^{\infty}}:=\max _{1 \leq m \leq M_{3}-1,1 \leq j \leq M_{2}-1,1 \leq i \leq M_{1}-1}\left|U_{i j m}^{N}-u_{i j m}^{N}\right|
$$

Example 1 We consider

$$
\begin{aligned}
& \int_{1}^{2} \Gamma(4-\beta)_{0}^{C} D_{t}^{\beta} u(x, y, t) d \beta \\
& \quad=\frac{\partial^{2} u(x, y, t)}{\partial x^{2}}+\frac{\partial^{2} u(x, y, t)}{\partial y^{2}}+\sin x \sin y\left[2\left(t^{3}+2 t+4\right)+\frac{6 t^{2}-6 t}{\ln t}\right] \\
& \quad-\left(t^{3}+2 t+4\right)^{2} \sin ^{2} x \sin ^{2} y+u^{2}(x, y, t), \\
& \quad 0<t \leq 1 / 2,(x, y) \in \Omega=(0, \pi) \times(0, \pi), \\
& u(x, y, t)=0, \quad(x, y) \in \partial \Omega, 0 \leq t \leq 1 / 2, \\
& u(x, y, 0)=4 \sin x \sin y, \quad u_{t}(x, y, 0)=2 \sin x \sin y, \quad(x, y) \in \Omega,
\end{aligned}
$$

whose analytical solution is known and is given by

$$
u(x, y, t)=\left(t^{3}+2 t+4\right) \sin x \sin y .
$$

A comparison of the numerical and exact solutions for Example 1 is presented in Fig. 1. It can be seen that they agree well with each other.

Table 1 records the numerical accuracy of scheme (43)-(44) and scheme (45)-(46) in time for Example 1. Let the step sizes $h_{1}, h_{2}$, and $\Delta \beta$ be fixed and small enough such that the dominating error arises from the approximation of the time derivatives. In the current test, the fixed step sizes are set as $h_{1}=h_{2}=\pi / 500, \Delta \beta=1 / 160$ for scheme (43)-(44), and $h_{1}=h_{2}=\pi / 100, \Delta \beta=1 / 10$ for scheme (45)-(46), respectively. Varying the step size in time, the numerical errors in the discrete $L^{2}$ norm and the associated convergence orders are shown in this table, which can be found in agreement with the theoretical analysis.

In Table 2, the fixed and small enough step sizes in space are taken, and an optimal step size ratio in time and distributed order is adopted for difference scheme (43)-(44). As $\Delta \beta$ and $\tau$ vary, we compute the errors and convergence orders listed in the table, which indicate that the convergence orders in time and distributed order are about one and two, respectively. It is in accord with the theoretical analysis.

Tables 3 and 4 display the computed results for Example 1 by the difference scheme (43)(44) and scheme (45)-(46) with an optimal step size ratio in time, space and distributed 


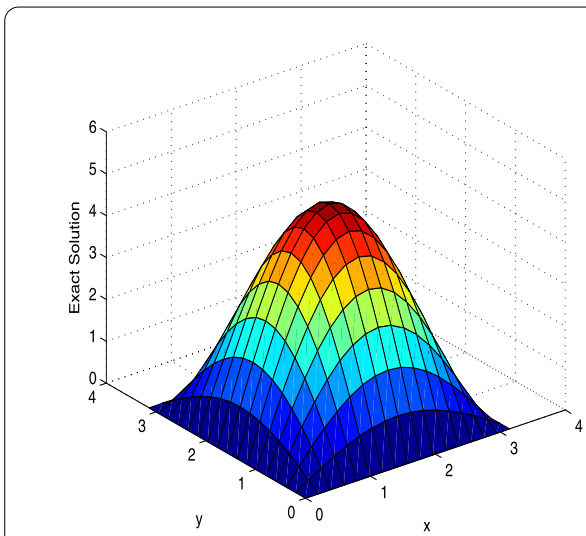

(a)

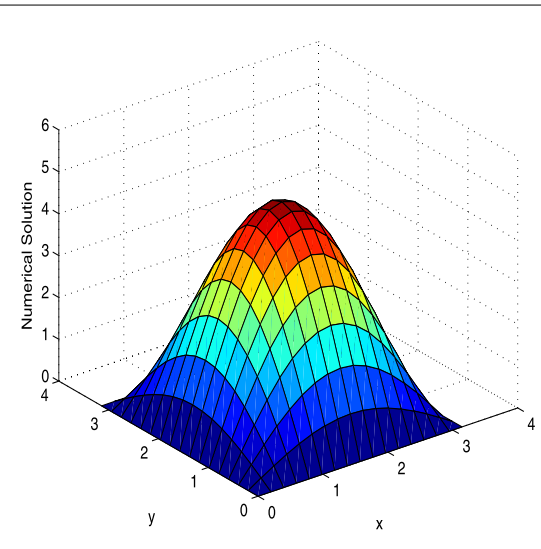

(b)

Figure 1 Exact solution (a) and numerical solution (b) at $T=0.5$ with mesh $h_{1}=h_{2}=\frac{\pi}{64}, \Delta \beta=\frac{1}{64}$ and $\tau=\frac{1}{4096}$ by scheme (43)-(44) (Example 1)

Table 1 Errors and convergence orders of scheme (43)-(44) and scheme (45)-(46) in temporal direction (Example 1)

\begin{tabular}{llllllll}
\hline$\tau$ & \multicolumn{2}{l}{ Scheme (43)-(44) } & & \multicolumn{3}{l}{ Scheme (45)-(46) } \\
\cline { 2 - 3 } & $\left\|e^{N}\right\|_{L^{2}}$ & Order & CPU time & & $\left\|e^{N}\right\|_{L^{2}}$ & Order & CPU time \\
\hline $1 / 10$ & 0.1225 & - & $0.1882 \mathrm{~s}$ & & 0.1222 & - & $0.0129 \mathrm{~s}$ \\
$1 / 20$ & 0.0634 & 0.9502 & $0.4328 \mathrm{~s}$ & & 0.0632 & 0.9512 & $0.0411 \mathrm{~s}$ \\
$1 / 40$ & 0.0326 & 0.9596 & $1.0168 \mathrm{~s}$ & & 0.0324 & 0.9639 & $0.0580 \mathrm{~s}$ \\
$1 / 80$ & 0.0167 & 0.9650 & $2.6598 \mathrm{~s}$ & & 0.0164 & 0.9823 & $0.1016 \mathrm{~s}$ \\
$1 / 160$ & 0.0085 & 0.9743 & $8.4336 \mathrm{~s}$ & & 0.0082 & 1.0000 & $0.2387 \mathrm{~s}$ \\
\hline
\end{tabular}

Table 2 Errors and convergence orders of scheme (43)-(44) with $h_{1}=h_{2}=\frac{\pi}{500}$, and an optimal step size ratio for $\tau$ and $\Delta \beta$ (Example 1)

\begin{tabular}{lllllll}
\hline$\tau$ & $\Delta \beta$ & $\left\|e^{N}\right\|_{L^{2}}$ & Order & $\left\|e^{N}\right\|_{L \infty}$ & Order & CPU time \\
\hline $1 / 100$ & $1 / 10$ & 0.0133 & - & 0.0093 & - & $3.7889 \mathrm{~s}$ \\
$1 / 400$ & $1 / 20$ & 0.0034 & 1.9678 & 0.0024 & 1.9542 & $44.8796 \mathrm{~s}$ \\
$1 / 1600$ & $1 / 40$ & $8.6411 \mathrm{e}-04$ & 1.9762 & $6.0481 \mathrm{e}-04$ & 1.9885 & $654.4187 \mathrm{~s}$ \\
$1 / 6400$ & $1 / 80$ & $2.1076 \mathrm{e}-04$ & 2.0365 & $1.4751 \mathrm{e}-04$ & 2.0357 & $2 \mathrm{~h} \mathrm{48} \mathrm{m} \mathrm{8} \mathrm{s}$ \\
\hline
\end{tabular}

Table 3 Errors and convergence orders of scheme (43)-(44) with an optimal step size ratio for $\tau, h_{1}$, $h_{2}$ and $\Delta \beta$ (Example 1)

\begin{tabular}{|c|c|c|c|c|c|c|c|}
\hline $\bar{\tau}$ & $h_{1}=h_{2}$ & $\Delta \beta$ & $\left\|e^{N}\right\|_{L^{2}}$ & Order & $\left\|e^{N}\right\|_{L \infty}$ & Order & CPU time \\
\hline $1 / 64$ & $\pi / 2$ & $1 / 8$ & 0.7230 & - & 0.4602 & - & $0.0816 \mathrm{~s}$ \\
\hline $1 / 256$ & $\pi / 4$ & $1 / 16$ & 0.1689 & 2.0978 & 0.1195 & 1.9453 & $0.0483 \mathrm{~s}$ \\
\hline $1 / 1024$ & $\pi / 8$ & $1 / 32$ & 0.0426 & 1.9872 & 0.0301 & 1.9892 & $0.2603 \mathrm{~s}$ \\
\hline $1 / 4096$ & $\pi / 16$ & $1 / 64$ & 0.0107 & 1.9932 & 0.0075 & 2.0048 & $5.4613 \mathrm{~s}$ \\
\hline $1 / 16,384$ & $\pi / 32$ & $1 / 128$ & 0.0027 & 1.9866 & 0.0019 & 1.9809 & $166.2940 \mathrm{~s}$ \\
\hline $1 / 65,536$ & $\pi / 64$ & $1 / 256$ & $6.6801 e-04$ & 2.0150 & $4.7098 \mathrm{e}-04$ & 2.0123 & $1 \mathrm{~h} 46 \mathrm{~m} 27 \mathrm{~s}$ \\
\hline
\end{tabular}

Table 4 Errors and convergence orders of scheme (45)-(46) with an optimal step size ratio for $\tau, h_{1}$, $h_{2}$ and $\Delta \beta$ (Example 1)

\begin{tabular}{lllllllr}
\hline$\tau$ & $h_{1}=h_{2}$ & $\Delta \beta$ & $\left\|e^{N}\right\|_{L^{2}}$ & Order & $\left\|e^{N}\right\|_{L \infty}$ & Order & CPU time \\
\hline $1 / 16$ & $\pi / 4$ & $1 / 2$ & 0.0913 & - & 0.0641 & - & $0.0234 \mathrm{~s}$ \\
$1 / 256$ & $\pi / 16$ & $1 / 4$ & 0.0056 & 4.0271 & 0.0040 & 4.0023 & $0.0729 \mathrm{~s}$ \\
$1 / 4096$ & $\pi / 64$ & $1 / 8$ & $3.3948 \mathrm{e}-04$ & 4.0440 & $2.4093 \mathrm{e}-04$ & 4.0533 & $30.6810 \mathrm{~s}$ \\
\hline
\end{tabular}


order, respectively. One can conclude from Table 3 that the convergence orders of scheme (43)-(44) with respect to time, space and distributed order are about one, two and two. While in Table 4, it can be found that the convergence orders of scheme (45)-(46) in time, space and distributed order are approximately one, two and four. These results agree well with the theoretical convergence orders.

Example 2 We now consider

$$
\begin{aligned}
& \int_{1}^{2} \Gamma(4-\beta)_{0}^{C} D_{t}^{\beta} u(x, y, z, t) d \beta \\
& \quad=\frac{\partial^{2} u(x, y, z, t)}{\partial x^{2}}+\frac{\partial^{2} u(x, y, z, t)}{\partial y^{2}}+\frac{\partial^{2} u(x, y, z, t)}{\partial z^{2}} \\
& \quad+\sin x \sin y \sin z\left[3\left(t^{3}+2 t+4\right)+\frac{6 t^{2}-6 t}{\ln t}\right] \\
& \quad-\left(t^{3}+2 t+4\right)^{2} \sin ^{2} x \sin ^{2} y \sin ^{2} z+u^{2}(x, y, z, t), \\
& \quad 0<t \leq 1 / 2,(x, y, z) \in \Omega=(0, \pi) \times(0, \pi) \times(0, \pi), \\
& u(x, y, z, t)=0, \quad(x, y, z) \in \partial \Omega, 0 \leq t \leq 1 / 2, \\
& u(x, y, z, 0)=4 \sin x \sin y \sin z, \quad u_{t}(x, y, z, 0)=2 \sin x \sin y \sin z, \quad(x, y, z) \in \Omega,
\end{aligned}
$$

whose analytical solution is known and is given by

$$
u(x, y, z, t)=\left(t^{3}+2 t+4\right) \sin x \sin y \sin z .
$$

Comparisons of numerical solutions with the exact solutions for Example 2 are shown in Figs. 2 and 3. In Fig. 2(a), let $y=z=\frac{\pi}{2}$. Then the numerical and exact solutions along the $x$-axis for different time instances are plotted with mesh $h_{1}=h_{2}=h_{3}=\frac{\pi}{32}, \Delta \beta=\frac{1}{32}$, and $\tau=\frac{1}{1024}$. Based on the same partition, fixing $x=\frac{\pi}{4}, z=\frac{3 \pi}{4}$, the numerical and exact solutions along the $y$-axis at different times are plotted in Fig. 2(b). Similarly, taking $x=\frac{\pi}{16}$, $y=\frac{3 \pi}{4}$, the numerical and exact solutions are drawn at different times along the $z$-axis in Fig. 2(c). Figure 3 presents the graphs of isosurfaces of the numerical solution (red) and exact solution (blue) when three isovalues are assigned with mesh $h_{1}=h_{2}=h_{3}=\frac{\pi}{50}$, $\Delta \beta=\frac{1}{20}$, and $\tau=\frac{1}{200}$. It can be seen intuitively from all these figures that the numerical solution is highly consistent with the exact solution, which indicates the reliability of the algorithm (59)-(61) for the three-dimensional problem.

In Table 5 , taking the step sizes $h_{1}, h_{2}, h_{3}$ and $\Delta \beta$ as fixed and small enough, then varying the step sizes in time, the numerical errors in the discrete $L^{2}$ norm and the associated convergence orders are computed and illustrated. For scheme (59)-(61), the fixed step sizes are assigned as $h_{1}=h_{2}=h_{3}=\pi / 100$ and $\Delta \beta=1 / 160$. When scheme (62)-(64) is employed, the step sizes in space and distributed order are $h_{1}=h_{2}=h_{3}=\pi / 50$ and $\Delta \beta=$ $1 / 10$, respectively. This table shows numerical accuracy of difference schemes (59)-(61) and (62)-(64) in time, which agrees well with the theoretical results.

In Tables 6 and 7, the algorithms (59)-(61) and (62)-(64) for Example 2 are tested further. The discrete $L^{2}$ and $L^{\infty}$ norms errors are given by taking an optimal step size ratio 


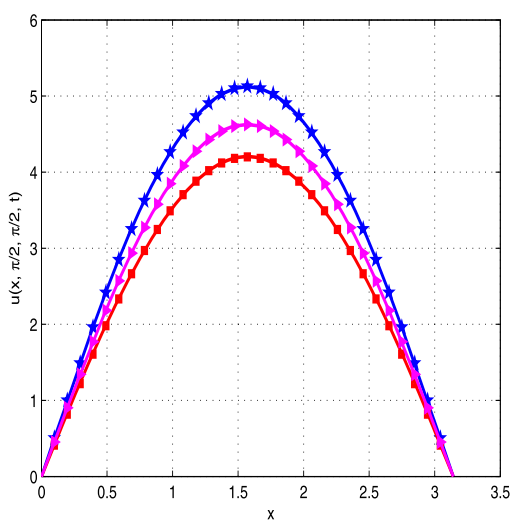

(a)

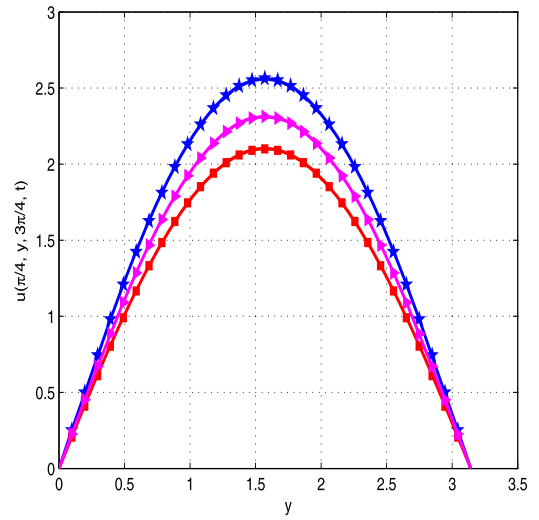

(b)

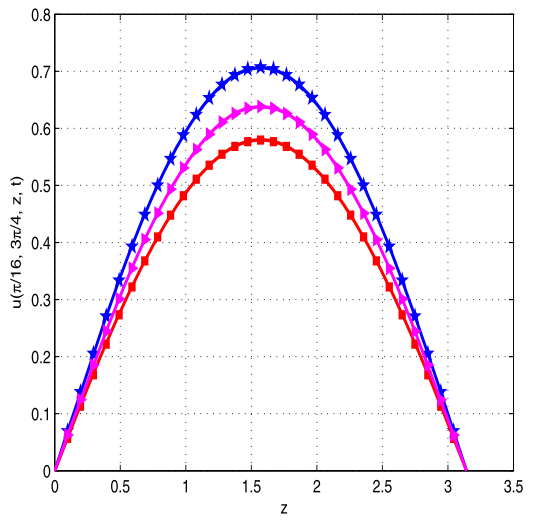

(c)

Figure 2 Exact solutions (lines) and numerical solutions (symbols) at $t=0.1$ (red), $t=0.3$ (magenta) and $t=0.5$ (blue) by using scheme (59)-(61) (Example 2)

in time, space and distributed order. This shows that the convergence orders of scheme (59)-(61) with respect to time, space and distributed order are approximately one, two and two, and that of scheme (62)-(64) are about one, two and four, respectively. The numerical results are in good agreement with the theoretical analysis, which demonstrates the effectiveness of the proposed methods for the three-dimensional problem.

Example 3 We consider

$$
\begin{aligned}
& \int_{1}^{2} 5_{0}^{\beta C} D_{t}^{\beta} u(x, y, z, t) d \beta \\
& \quad=\frac{\partial^{2} u(x, y, z, t)}{\partial x^{2}}+\frac{\partial^{2} u(x, y, z, t)}{\partial y^{2}}+\frac{\partial^{2} u(x, y, z, t)}{\partial z^{2}} \\
& \quad 0<t \leq 1,(x, y, z) \in \Omega=(0,1) \times(0,1) \times(0,1), \\
& u(x, y, z, t)=0, \quad(x, y, z) \in \partial \Omega, 0 \leq t \leq 1, \\
& u(x, y, z, 0)=x^{2}\left(1-x^{2}\right) y^{2}\left(1-y^{2}\right) z^{2}\left(1-z^{2}\right), \quad u_{t}(x, y, z, 0)=0, \quad(x, y, z) \in \Omega .
\end{aligned}
$$



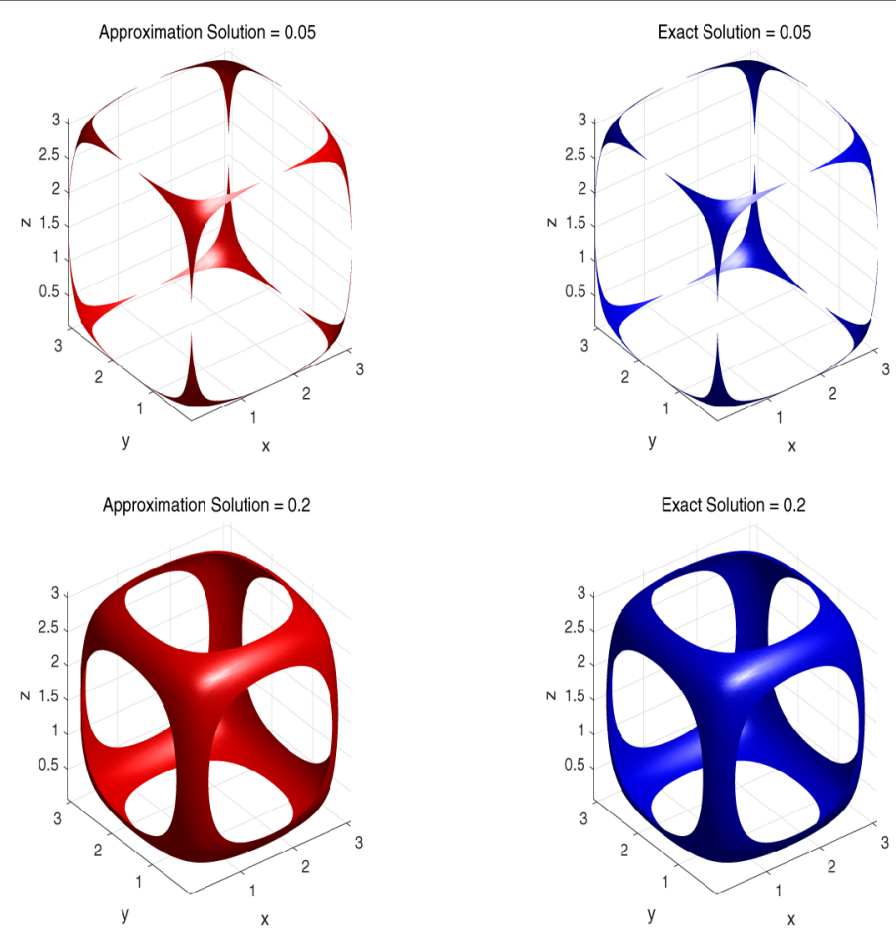

Approximation Solution $=3.6$

Exact Solution $=3.6$
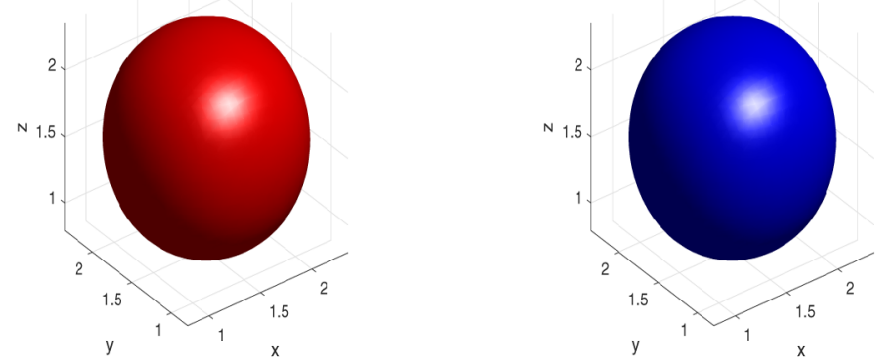

Figure 3 The graphs of isosurfaces for the approximation solution (red) and exact solution (blue) with different isovalues by scheme (59)-(61) (Example 2)

Table 5 Errors and convergence orders of scheme (59)-(61) and scheme (62)-(64) in the temporal direction (Example 2)

\begin{tabular}{llllllll}
\hline$\tau$ & \multicolumn{2}{l}{ Scheme (59)-(61) } & & \multicolumn{3}{l}{ Scheme (62)-(64) } \\
\cline { 2 - 3 } & $\left\|e^{N}\right\|_{L^{2}}$ & Order & CPU time & & $\left\|e^{N}\right\|_{L^{2}}$ & Order & CPU time \\
\hline $1 / 10$ & 0.1869 & - & $0.9506 \mathrm{~s}$ & & 0.1855 & - & $0.1057 \mathrm{~s}$ \\
$1 / 20$ & 0.0958 & 0.9642 & $2.0467 \mathrm{~s}$ & & 0.0944 & 0.9746 & $0.1882 \mathrm{~s}$ \\
$1 / 40$ & 0.0488 & 0.9731 & $4.8517 \mathrm{~s}$ & & 0.0474 & 0.9939 & $0.3908 \mathrm{~s}$ \\
$1 / 80$ & 0.0246 & 0.9882 & $12.9781 \mathrm{~s}$ & & 0.0232 & 1.0308 & $0.7970 \mathrm{~s}$ \\
$1 / 160$ & 0.0123 & 1.0000 & $39.2808 \mathrm{~s}$ & & 0.0108 & 1.1031 & $2.1005 \mathrm{~s}$ \\
\hline
\end{tabular}

Table 6 Errors and convergence orders of scheme (59)-(61) with an optimal step size ratio for $\tau, h_{1}$, $h_{2}, h_{3}$ and $\Delta \beta$ (Example 2)

\begin{tabular}{lllllllr}
\hline$\tau$ & $h_{1}=h_{2}=h_{3}$ & $\Delta \beta$ & $\left\|e^{N}\right\|_{L^{2}}$ & Order & $\left\|e^{N}\right\|_{L} \infty$ & Order & CPU time \\
\hline $1 / 16$ & $\pi / 4$ & $1 / 4$ & 0.1717 & - & 0.0998 & - & $0.0285 \mathrm{~s}$ \\
$1 / 64$ & $\pi / 8$ & $1 / 8$ & 0.0435 & 1.9808 & 0.0256 & 1.9629 & $0.0348 \mathrm{~s}$ \\
$1 / 256$ & $\pi / 16$ & $1 / 16$ & 0.0108 & 2.0100 & 0.0064 & 2.0000 & $0.2327 \mathrm{~s}$ \\
$1 / 1024$ & $\pi / 32$ & $1 / 32$ & 0.0027 & 2.0000 & 0.0016 & 2.0000 & $14.0054 \mathrm{~s}$ \\
\hline
\end{tabular}


Table 7 Errors and convergence orders of scheme (62)-(64) with an optimal step size ratio for $\tau, h_{1}$, $h_{2}, h_{3}$ and $\Delta \beta$ (Example 2)

\begin{tabular}{llllllll}
\hline$\tau$ & $h_{1}=h_{2}$ & $\Delta \beta$ & $\left\|e^{N}\right\|_{L^{2}}$ & Order & $\left\|e^{N}\right\|_{L} \infty$ & Order & CPU time \\
\hline $1 / 16$ & $\pi / 4$ & $1 / 2$ & 0.1708 & - & 0.0992 & - & $0.0033 \mathrm{~s}$ \\
$1 / 256$ & $\pi / 16$ & $1 / 4$ & 0.0107 & 3.9966 & 0.0063 & 3.9769 & $0.2109 \mathrm{~s}$ \\
$1 / 4096$ & $\pi / 64$ & $1 / 8$ & $6.5768 \mathrm{e}-04$ & 4.0241 & $3.8735 \mathrm{e}-04$ & 4.0236 & $1 \mathrm{~h} 6 \mathrm{~m} \mathrm{37 \textrm {s }}$ \\
\hline
\end{tabular}

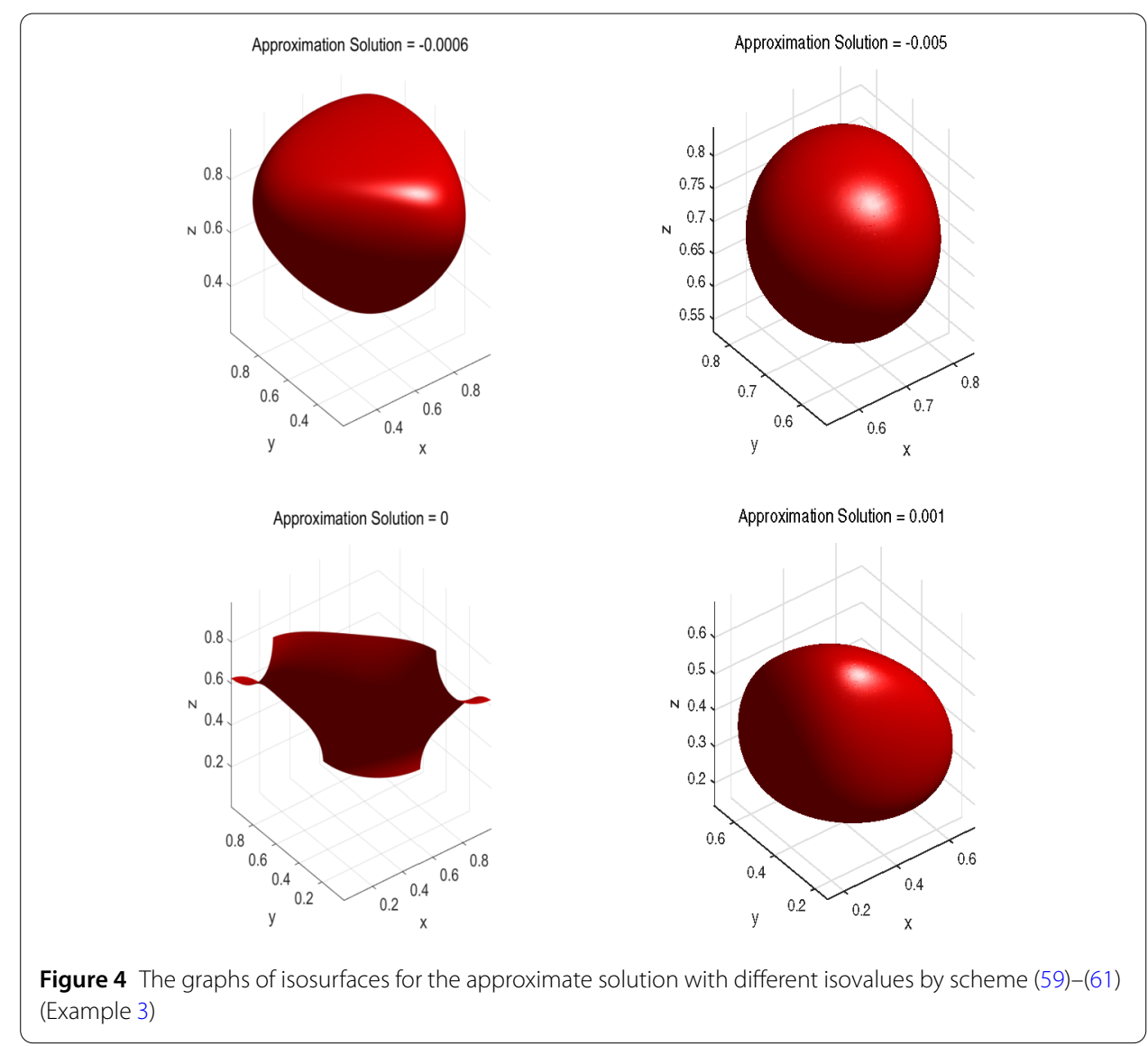

In the current example, a three-dimensional distributed-order wave equation with a zero source term is considered. Figure 4 presents the graphs of isosurfaces of the numerical solution with mesh $h_{1}=h_{2}=h_{3}=\frac{1}{100}, \Delta \beta=\frac{1}{50}$, and $\tau=\frac{1}{200}$ at the final time $T=1$.

\section{Conclusion}

In this paper, based on two quadrature rules, two ADI difference schemes are established for two- and three-dimensional time-fractional wave equation of distributed-order, where a nonlinear source term is considered and handled. As a representative, we provide the theoretical analysis on unique solvability, unconditional stability and convergence for the two-dimensional case by the discrete energy method. Numerical results show that the proposed methods are feasible and effective. Moreover, comparisons between the two ADI schemes for two- and three-dimensional problems are reported, respectively. In a future work, we intend to investigate the higher order approaches for handling the nonlinear term and for approximating the time-fractional derivatives, so as to develop higher order schemes for solving the fractional problems with nonlinear terms. 


\section{Acknowledgements}

The authors would like to express their gratitude to the referees for their very helpful comments and suggestions on the manuscript.

\section{Funding}

This research was supported by the National Natural Science Foundation of China under grant 11471262.

\section{Competing interests}

The authors declare that they have no competing interests.

\section{Authors' contributions}

$\mathrm{JH}$ carried out the numerical algorithms. JW conceived of the study and analyzed the theoretical results. YN helped to draft the manuscript. All the authors read and approved the final manuscript.

\section{Author details}

'Research Center for Computational Science, Northwestern Polytechnical University, Xi'an, China. ${ }^{2}$ College of Science, Henan University of Technology, Zhengzhou, China.

\section{Publisher's Note}

Springer Nature remains neutral with regard to jurisdictional claims in published maps and institutional affiliations.

\section{Received: 24 January 2018 Accepted: 24 September 2018 Published online: 03 October 2018}

\section{References}

1. Abbaszadeh, M., Dehghan, M:: An improved meshless method for solving two-dimensional distributed order time-fractional diffusion-wave equation with error estimate. Numer. Algorithms 75(1), 173-211 (2016)

2. Atanackovic, T.M., Pilipovic, S., Zorica, D.: Time distributed-order diffusion-wave equation. I. Volterra-type equation. Proc. R. Soc. Lond., Ser. A, Math. Phys. Eng. Sci.. 465, 1869-1891 (2009)

3. Bhrawy, A.H., Zaky, M.A.: A method based on the Jacobi tau approximation for solving multi-term time-space fractional partial differential equations. J. Comput. Phys. 281, 876-895 (2015)

4. Bhrawy, A.H., Zaky, M.A.: Numerical simulation of multi-dimensional distributed-order generalized Schrödinger equations. Nonlinear Dyn. 89(2), 1415-1432 (2017)

5. Caputo, M.: Elasticità e dissipazione. Zanichelli, Bologna (1969)

6. Caputo, M.: Distributed order differential equations modelling dielectric induction and diffusion. Fract. Calc. Appl. Anal. 4(4), 421-442 (2001)

7. Chechkin, A.V., Gorenflo, R., Sokolov, I.M. Gonchar, V.Y.: Distributed order time fractional diffusion equation. Fract. Calc. Appl. Anal. 6(3), 259-280 (2003)

8. Chechkin, A.V., Klafter, J., Sokolov, I.M.: Fractional Fokker-Planck equation for ultraslow kinetics. Europhys. Lett. 63(3), 326-332 (2003)

9. Dehghan, M., Abbaszadeh, M.: Element free Galerkin approach based on the reproducing kernel particle method for solving 2D fractional Tricomi-type equation with Robin boundary condition. Comput. Math. Appl. 73(6), 1270-1285 (2017)

10. Diethelm, K., Ford, N.J.: Numerical analysis for distributed-order differential equations. J. Comput. Appl. Math. 225(1), 96-104 (2009)

11. Eab, C., Lim, S.: Fractional Langevin equations of distributed order. Phys. Rev. E 83(3), 031136 (2011)

12. Ford, N.J., Morgado, M.L., Rebelo, M.: An implicit finite difference approximation for the solution of the diffusion equation with distributed order in time. Electron. Trans. Numer. Anal. 44, 289-305 (2015)

13. Gao, G., Sun, H., Sun, Z:: Some high-order difference schemes for the distributed-order differential equations. J. Comput. Phys. 298, 337-359 (2015)

14. Gao, G., Sun, Z:: Two alternating direction implicit difference schemes with the extrapolation method for the two-dimensional distributed-order differential equations. Comput. Math. Appl. 69(9), 926-948 (2015)

15. Gao, G.s Sun, Z.: Two alternating direction implicit difference schemes for solving the two-dimensional time distributed-order wave equations. J. Sci. Comput. 69(2), 506-531 (2016)

16. Gao, G., Sun, Z.: Two alternating direction implicit difference schemes for two-dimensional distributed-order fractional diffusion equations. J. Sci. Comput. 66(3), 1281-1312 (2016)

17. Gao, G., Sun, Z:: Two unconditionally stable and convergent difference schemes with the extrapolation method for the one-dimensional distributed-order differential equations. Numer. Methods Partial Differ. Equ. 32(2), 591-615 (2016)

18. Gorenflo, R., Luchko, Y., Stojanović, M.: Fundamental solution of a distributed order time-fractional diffusion-wave equation as probability density. Fract. Calc. Appl. Anal. 16(2), 297-316 (2013)

19. Hartley, T.T., Lorenzo, C.F.: Fractional system identification: an approach using continuous order-distributions. NASA (1999)

20. Jiao, Z., Chen, Y., Podlubny, I.: Distributed-Order Dynamic Systems: Stability, Simulation, Applications and Perspectives. Springer Briefs in Electrical and Computer Engineering, pp. 90-97. Springer, London (2012)

21. Katsikadelis, J.T.: Numerical solution of distributed order fractional differential equations. J. Comput. Phys. 259, 11-22 (2014)

22. Kochubei, A.N.: Distributed order calculus and equations of ultraslow diffusion. J. Math. Anal. Appl. 340(1), 252-281 (2008)

23. Li, Z., Luchko, Y., Yamamoto, M.: Asymptotic estimates of solutions to initial-boundary-value problems for distributed order time-fractional diffusion equations. Fract. Calc. Appl. Anal. 17(4), 1114-1136 (2014) 
24. Morgado, M.L., Rebelo, M.: Numerical approximation of distributed order reaction-diffusion equations. J. Comput. Appl. Math. 275, 216-227 (2015)

25. Podlubny, I., Skovranek, T., Jara, B.M.V., Petras, I., Verbitsky, V., Chen, Y.: Matrix approach to discrete fractional calculus III: non-equidistant grids, variable step length and distributed orders. Philos. Trans. R. Soc. Lond. A 371(1990), 20120153 (2013)

26. Quarteroni, A. Valli, A.: Numerical Approximation of Partial Differential Equations. Springer Series in Computational Mathematics, vol. 23. Springer, Berlin (2008)

27. Rida, S., El-Sayed, A., Arafa, A.: On the solutions of time-fractional reaction-diffusion equations. Commun. Nonlinear Sci. Numer. Simul. 15(12), 3847-3854 (2010)

28. Samarskii, A., Andreev, V.: Difference Methods for Elliptic Equations. Nauka, Moscow (1976)

29. Sinai, Y.G.: The limiting behavior of a one-dimensional random walk in a random medium. Theory Probab. Appl. 27(2), 256-268 (1983)

30. Sun, Z: The Method of Order Reduction and Its Application to the Numerical Solutions of Partial Differential Equations. Science Press, Beijing (2009)

31. Wazwaz, A.M., Gorguis, A.: An analytic study of Fisher's equation by using Adomian decomposition method. Appl. Math. Comput. 154(3), 609-620 (2004)

32. Ye, H., Liu, F., Anh, V.: Compact difference scheme for distributed-order time-fractional diffusion-wave equation on bounded domains. J. Comput. Phys. 298, 652-660 (2015)

33. Zaky, M.A.: A Legendre spectral quadrature tau method for the multi-term time-fractional diffusion equations. Comput. Appl. Math. 37(3), 3525-3538 (2018)

34. Zaky, M.A.: A Legendre collocation method for distributed-order fractional optimal control problems. Nonlinear Dyn. 91(4), 2667-2681 (2018)

35. Zaky, M.A., Tenreiro Machado, J.A.: On the formulation and numerical simulation of distributed-order fractional optimal control problems. Commun. Nonlinear Sci. Numer. Simul. 52, 177-189 (2017)

\section{Submit your manuscript to a SpringerOpen ${ }^{\circ}$ journal and benefit from:}

- Convenient online submission

- Rigorous peer review

Open access: articles freely available online

- High visibility within the field

Retaining the copyright to your article

Submit your next manuscript at $\gg$ springeropen.com 